\title{
Exact finite-difference scheme and nonstandard finite-difference scheme for coupled Burgers equation
}

\author{
Lei Zhang, Lisha Wang and Xiaohua Ding*
}

${ }^{\text {"Correspondence: }}$ mathdxh@hit.edu.cn

Department of Mathematics, Harbin Institute of Technology at Weihai,

2 Wenhua West Road, Weihai, 264209, China

\begin{abstract}
This work develops exact finite-difference schemes for the two-dimensional nonlinear coupled viscous Burgers equation using the analytic solution. We extend the explicit nonstandard finite-difference schemes on the basis of the exact finite-difference schemes to solve the coupled Burgers equation. Numerical examples are presented to verify the efficiency and accuracy of the methods.
\end{abstract}

MSC: 39A10; 65L12; 74H 15

Keywords: coupled Burgers equation; exact finite-difference scheme; nonstandard finite-difference scheme

\section{Introduction}

The coupled viscous Burgers equation is a form of the Navier-Stokes equations having a known form for the exact solutions. It is a simple model of physical flows and problems and has become an important partial differential equation in fluid dynamics and various physical applications [1].

Fletcher [2] proposed the analytic solution of two-dimensional coupled Burgers equations using the Hopf-Cole transformation. And the numerical solutions of coupled Burgers equations have attracted considerable attention of many researchers [1,3-8]. Srivastava et al. [1, 5-7] gave the implicit finite-difference scheme, the Crank-Nicolson scheme, the semi-implicit finite-difference scheme, and the Crank-Nicolson semi-implicit scheme for the coupled Burgers equations. Bahadir [3] obtained a fully implicit finite-difference scheme to solve the coupled Burgers equations. Jain and Holla [4] developed two algorithms based on cubic spline method for the coupled Burgers equations. Kweyu et al. [8] gave an explicit scheme for the coupled Burgers equations under varied initial and boundary conditions.

Two-dimensional nonlinear coupled viscous Burgers equations can be expressed as

$$
\left\{\begin{array}{l}
u_{t}+u u_{x}+v u_{y}=\frac{1}{R}\left(u_{x x}+u_{y y}\right), \\
v_{t}+u v_{x}+v v_{y}=\frac{1}{R}\left(v_{x x}+v_{y y}\right),
\end{array}\right.
$$

subject to the initial conditions

$$
\begin{cases}u(x, y, 0)=f(x, y), & (x, y) \in \Omega, \\ v(x, y, 0)=g(x, y), & (x, y) \in \Omega,\end{cases}
$$

\section{Springer}

O2014 Zhang et al.; licensee Springer. This is an Open Access article distributed under the terms of the Creative Commons Attribution License (http://creativecommons.org/licenses/by/2.0), which permits unrestricted use, distribution, and reproduction in any medium, provided the original work is properly cited. 
and the boundary conditions

$$
\begin{cases}u(x, y, t)=f_{1}(x, y, t), & x, y \in \partial \Omega, t>0, \\ v(x, y, t)=g_{1}(x, y, t), & x, y \in \partial \Omega, t>0\end{cases}
$$

where $\Omega=\{(x, y): a \leq x \leq b, a \leq y \leq b\}, \partial \Omega$ is its boundary, $u(x, y, t)$ and $v(x, y, t)$ are the velocity components to be determined, $f, g, f_{1}, g_{1}$ are known functions, and $R$ is the Reynolds number. Among various techniques for solving the coupled Burgers equation, the nonstandard finite-difference (NSFD) schemes have been proved to be one of the most efficient approaches in recent years. Compared with some other methods, NSFD method is more stable $[1,3,5]$. But until now, no researchers have given the exact finite-difference scheme and given the nonstandard finite difference based on the exact finite-difference scheme for the coupled Burgers (1).

The main purpose of this paper is to give an exact finite-difference scheme for the coupled viscous Burgers equations (1) using the analytic solution and give the explicit nonstandard finite-difference schemes on the basis of the exact finite-difference schemes. Two classic examples will be presented and the results illustrate the efficiency and accuracy of our methods.

The remainder of the paper is organized as follows. In the next section, exact difference schemes are proposed for solving (1). Then Section 3 gives two explicit nonstandard finite-difference schemes on the basis of the exact finite-difference schemes in Section 2. Numerical experiments and analyses are given in Section 4.

\section{Analysis of exact finite-difference scheme for coupled Burgers equation}

An analytic solution of Burgers equations (1) was given by Fletcher [2] using the Hopf-Cole transformation as follows:

$$
\left\{\begin{array}{l}
u(x, y, t)=\frac{3}{4}-\frac{1}{4\left(1+e^{\frac{R}{32}(4 y-4 x-t)}\right)}, \\
v(x, y, t)=\frac{3}{4}+\frac{1}{4\left(1+e^{\frac{R}{32}(4 y-4 x-t)}\right)} .
\end{array}\right.
$$

From the analytic solution (2), we obtain $v(x, y, t)=\frac{3}{2}-u(x, y, t)$. If $4 \Delta x=4 \Delta y=\Delta t$, we have $u(x+\Delta x, y, t)=u(x, y-\Delta y, t)=u(x, y, t+\Delta t)$ and $v(x+\Delta x, y, t)=v(x, y-\Delta y, t)=$ $v(x, y, t+\Delta t)$. Because the analysis process is very long, we divide it into two parts for the readers' convenience.

First, considering the first equation of (2), we have

$$
\frac{1}{\frac{3}{4}-u(x, y, t)}=4\left(1+e^{\frac{R}{32}(4 y-4 x-t)}\right)
$$

and

$$
\begin{aligned}
& \frac{1}{\frac{3}{4}-u(x+\Delta x, y, t)}=4\left(1+e^{-\frac{R}{8} \Delta x} e^{\frac{R}{22}(4 y-4 x-t)}\right), \\
& \frac{1}{\frac{3}{4}-u(x-\Delta x, y, t)}=4\left(1+e^{\frac{R}{8} \Delta x} e^{\frac{R}{32}(4 y-4 x-t)}\right), \\
& \frac{1}{\frac{3}{4}-u(x, y-\Delta y, t)}=4\left(1+e^{-\frac{R}{8} \Delta y} e^{\frac{R}{32}(4 y-4 x-t)}\right),
\end{aligned}
$$




$$
\frac{1}{\frac{3}{4}-u(x, y+\Delta y, t)}=4\left(1+e^{\frac{R}{8} \Delta y} e^{\frac{R}{32}(4 y-4 x-t)}\right) .
$$

Now for the second equation of (2), we can also get

$$
\frac{1}{v(x, y, t)-\frac{3}{4}}=4\left(1+e^{\frac{R}{32}(4 y-4 x-t)}\right)
$$

and

$$
\begin{aligned}
& \frac{1}{v(x+\Delta x, y, t)-\frac{3}{4}}=4\left(1+e^{-\frac{R}{8} \Delta x} e^{\frac{R}{32}(4 y-4 x-t)}\right), \\
& \frac{1}{v(x-\Delta x, y, t)-\frac{3}{4}}=4\left(1+e^{\frac{R}{8} \Delta x} e^{\frac{R}{32}(4 y-4 x-t)}\right), \\
& \frac{1}{v(x, y-\Delta y, t)-\frac{3}{4}}=4\left(1+e^{-\frac{R}{8} \Delta y} e^{\frac{R}{32}(4 y-4 x-t)}\right), \\
& \frac{1}{v(x, y+\Delta y, t)-\frac{3}{4}}=4\left(1+e^{\frac{R}{8} \Delta y} e^{\frac{R}{32}(4 y-4 x-t)}\right) .
\end{aligned}
$$

We will do some appropriate transformation to (3) and (4) in the following analysis.

\subsection{Explicit exact-difference scheme}

In this section, we will give the explicit finite-difference scheme for (1).

\subsubsection{Discrete scheme for the first equation}

Using (3a) minus (3), we obtain

$$
\begin{aligned}
\frac{1}{\frac{3}{4}-u(x+\Delta x, y, t)}-\frac{1}{\frac{3}{4}-u(x, y, t)} & =\left(e^{-\frac{R}{8} \Delta x}-1\right) 4 e^{\frac{R}{32}(4 y-4 x-t)} \\
& =\left(e^{-\frac{R}{8} \Delta x}-1\right)\left(\frac{1}{\frac{3}{4}-u(x, y, t)}-4\right) .
\end{aligned}
$$

By defining $\phi_{1}=\left(1-e^{-\frac{R}{8} \Delta x}\right) /\left(\frac{R}{8}\right)$, using (5), we get the following result:

$$
\frac{u(x+\Delta x, y, t)-u(x, y, t)}{\phi_{1}}=\frac{R}{4}\left(\frac{3}{4}-u(x+\Delta x, y, t)\right)(1-2 u(x, y, t)) .
$$

If we set $u_{x x}=\frac{u(x+\Delta x, y, t)-2 u(x, y, t)+u(x-\Delta x, y, t)}{\phi_{1} \phi_{2}}$ and use (6), we obtain

$$
\begin{aligned}
\frac{1}{R} u_{x x} & =\frac{u(x+\Delta x, y, t)-2 u(x, y, t)+u(x-\Delta x, y, t)}{R \phi_{1} \phi_{2}} \\
& =\frac{\frac{u(x+\Delta x, y, t)-u(x, y, t)}{\phi_{1}}-\frac{u(x, y, t)-u(x-\Delta x, y, t)}{\phi_{1}}}{R \phi_{2}} \\
& =\frac{\frac{R}{4}\left(\frac{3}{4}-u(x+\Delta x, y, t)\right)(1-2 u(x, y, t))-\frac{R}{4}\left(\frac{3}{4}-u(x, y, t)\right)(1-2 u(x-\Delta x, y, t))}{R \phi_{2}} \\
& =\frac{1}{4} \frac{\left(\frac{3}{4}-u(x+\Delta x, y, t)\right)(1-2 u(x, y, t))-\left(\frac{3}{4}-u(x, y, t)\right)(1-2 u(x-\Delta x, y, t))}{\phi_{2}}
\end{aligned}
$$




$$
\begin{aligned}
= & \frac{u(x, y, t)-u(x+\Delta x, y, t)}{4 \phi_{2}}-\frac{3}{2} \frac{u(x, y, t)-u(x-\Delta x, y, t)}{4 \phi_{2}} \\
& +u(x, y, t) \frac{u(x+\Delta x, y, t)-u(x-\Delta x, y, t)}{2 \phi_{2}} .
\end{aligned}
$$

And if we use (3) minus equation (3c), we get

$$
\begin{aligned}
\frac{1}{\frac{3}{4}-u(x, y, t)}-\frac{1}{\frac{3}{4}-u(x, y-\Delta y, t)} & =\left(1-e^{-\frac{R}{8} \Delta y}\right) 4 e^{\frac{R}{32}(4 y-4 x-t)} \\
& =\left(1-e^{-\frac{R}{8} \Delta y}\right)\left(\frac{1}{\frac{3}{4}-u(x, y, t)}-4\right) .
\end{aligned}
$$

Setting $\varphi_{2}=\left(1-e^{-\frac{R}{8} \Delta y}\right) /\left(\frac{R}{8}\right)$, we immediately obtain

$$
\frac{u(x, y, t)-u(x, y-\Delta y, t)}{\varphi_{2}}=\frac{R}{4}\left(\frac{3}{4}-u(x, y-\Delta y, t)\right)(2 u(x, y, t)-1)
$$

According to (9), if we define $u_{y y}=\frac{u(x, y+\Delta y, t)-2 u(x, y, t)+u(x, y-\Delta y, t)}{\varphi_{1} \varphi_{2}}$, we have

$$
\begin{aligned}
\frac{1}{R} u_{y y}= & \frac{u(x, y+\Delta y, t)-2 u(x, y, t)+u(x, y-\Delta y, t)}{R \varphi_{1} \varphi_{2}} \\
= & \frac{\frac{u(x, y+\Delta y, t)-u(x, y, t)}{\varphi_{2}}-\frac{u(x, y, t)-u(x, y-\Delta y, t)}{\varphi_{2}}}{R \varphi_{1}} \\
= & \frac{\frac{R}{4}\left(\frac{3}{4}-u(x, y, t)\right)(2 u(x, y+\Delta y, t)-1)-\frac{R}{4}\left(\frac{3}{4}-u(x, y-\Delta y, t)\right)(2 u(x, y, t)-1)}{R \varphi_{1}} \\
= & \frac{1}{4} \frac{\left(\frac{3}{4}-u(x, y, t)\right)(2 u(x, y+\Delta y, t)-1)-\left(\frac{3}{4}-u(x, y-\Delta y, t)\right)(2 u(x, y, t)-1)}{\varphi_{1}} \\
= & \frac{u(x, y, t)-u(x, y-\Delta y, t)}{4 \varphi_{1}}+\frac{3}{2} \frac{u(x, y+\Delta y, t)-u(x, y, t)}{4 \varphi_{1}} \\
& -u(x, y, t) \frac{u(x, y+\Delta y, t)-u(x, y-\Delta y, t)}{2 \varphi_{1}} .
\end{aligned}
$$

Based on (7) and (10), we have the following result:

$$
\begin{aligned}
\frac{1}{R} u_{x x}+\frac{1}{R} u_{y y}= & \frac{u(x, y, t)-u(x+\Delta x, y, t)}{4 \phi_{2}}-\frac{3}{2} \frac{u(x, y, t)-u(x-\Delta x, y, t)}{4 \phi_{2}} \\
& +u(x, y, t) \frac{u(x+\Delta x, y, t)-u(x-\Delta x, y, t)}{2 \phi_{2}}+\frac{u(x, y, t)-u(x, y-\Delta y, t)}{4 \varphi_{1}} \\
& +\frac{3}{2} \frac{u(x, y+\Delta y, t)-u(x, y, t)}{4 \varphi_{1}}-u(x, y, t) \frac{u(x, y+\Delta y, t)-u(x, y-\Delta y, t)}{2 \varphi_{1}} \\
= & \frac{u(x, y, t)-u(x, y-\Delta y, t)}{4 \phi_{2}}+\frac{3}{2} \frac{u(x, y+\Delta y, t)-u(x, y, t)}{4 \phi_{2}} \\
& +u(x, y, t) \frac{u(x+\Delta x, y, t)-u(x-\Delta x, y, t)}{2 \phi_{2}}+\frac{u(x, y, t)-u(x, y-\Delta y, t)}{4 \varphi_{1}} \\
& +\frac{3}{2} \frac{u(x, y+\Delta y, t)-u(x, y, t)}{4 \varphi_{1}}-u(x, y, t) \frac{u(x, y+\Delta y, t)-u(x, y-\Delta y, t)}{2 \varphi_{1}}
\end{aligned}
$$




$$
\begin{aligned}
= & \frac{3}{2} \frac{u(x, y, t)-u(x, y-\Delta y, t)}{4 \phi_{2}}-\frac{1}{2} \frac{u(x, y, t)-u(x, y-\Delta y, t)}{4 \phi_{2}} \\
& +\frac{3}{2} \frac{u(x, y+\Delta y, t)-u(x, y, t)}{4 \phi_{2}}+u(x, y, t) \frac{u(x+\Delta x, y, t)-u(x-\Delta x, y, t)}{2 \phi_{2}} \\
& +\frac{3}{2} \frac{u(x, y, t)-u(x, y-\Delta y, t)}{4 \varphi_{1}}-\frac{1}{2} \frac{u(x, y, t)-u(x, y-\Delta y, t)}{4 \varphi_{1}} \\
& +\frac{3}{2} \frac{u(x, y+\Delta y, t)-u(x, y, t)}{4 \varphi_{1}}-u(x, y, t) \frac{u(x, y+\Delta y, t)-u(x, y-\Delta y, t)}{2 \varphi_{1}} \\
= & \frac{3}{2} \frac{u(x, y+\Delta y, t)-u(x, y-\Delta y, t)}{4 \phi_{2}}-\frac{1}{2} \frac{u(x, y, t)-u(x, y-\Delta y, t)}{4 \phi_{2}} \\
& +u(x, y, t) \frac{u(x+\Delta x, y, t)-u(x-\Delta x, y, t)}{2 \phi_{2}} \\
& +\frac{3}{2} \frac{u(x, y+\Delta y, t)-u(x, y-\Delta y, t)}{4 \varphi_{1}}-\frac{1}{2} \frac{u(x, y, t)-u(x, y-\Delta y, t)}{4 \varphi_{1}} \\
& -u(x, y, t) \frac{u(x, y+\Delta y, t)-u(x, y-\Delta y, t)}{2 \varphi_{1}} .
\end{aligned}
$$

Because $4 \Delta x=4 \Delta y=\Delta t, \psi_{1}=\left(e^{\frac{R}{32} \Delta t}-1\right) /\left(\frac{R}{32}\right), \phi_{2}=\varphi_{1}$, we have

$$
\begin{aligned}
\frac{1}{R} u_{x x}+\frac{1}{R} u_{y y}= & u(x, y, t) \frac{u(x+\Delta x, y, t)-u(x-\Delta x, y, t)}{2 \phi_{2}} \\
& +\frac{3}{2} \frac{u(x, y+\Delta y, t)-u(x, y-\Delta y, t)}{2 \varphi_{1}}-\frac{u(x, y, t)-u(x, y-\Delta y, t)}{4 \varphi_{1}} \\
& -u(x, y, t) \frac{u(x, y+\Delta y, t)-u(x, y-\Delta y, t)}{2 \varphi_{1}} \\
= & u(x, y, t) \frac{u(x+\Delta x, y, t)-u(x-\Delta x, y, t)}{2 \phi_{2}}-\frac{u(x, y, t)-u(x, y, t+\Delta t)}{4 \varphi_{1}} \\
& +\left(\frac{3}{2}-u(x, y, t)\right) \frac{u(x, y+\Delta y, t)-u(x, y-\Delta y, t)}{2 \varphi_{1}} \\
= & u(x, y, t) \frac{u(x+\Delta x, y, t)-u(x-\Delta x, y, t)}{2 \phi_{2}}+\frac{u(x, y, t+\Delta t)-u(x, y, t)}{\psi_{1}} \\
& +v(x, y, t) \frac{u(x, y+\Delta y, t)-u(x, y-\Delta y, t)}{2 \varphi_{1}} \\
\doteq & u u_{x}+u_{t}+v u_{y} .
\end{aligned}
$$

Equation (12) can be seen as a discrete format of the first equation of (1). This discrete format is an explicit scheme. In the following section, we will consider the discrete scheme for the second equation of (1).

\subsubsection{Discrete scheme for the second equation}

Using (4a) minus (4), we obtain

$$
\begin{aligned}
\frac{1}{v(x+\Delta x, y, t)-\frac{3}{4}}-\frac{1}{v(x, y, t)-\frac{3}{4}} & =\left(e^{-\frac{R}{8} \Delta x}-1\right) 4 e^{\frac{R}{32}(4 y-4 x-t)} \\
& =\left(e^{-\frac{R}{8} \Delta x}-1\right)\left(\frac{1}{v(x, y, t)-\frac{3}{4}}-4\right) .
\end{aligned}
$$


Setting $\phi_{3}=\left(1-e^{-\frac{R}{8} \Delta x}\right) /\left(\frac{R}{8}\right)$, then according to (13) we have the following equation:

$$
\frac{v(x+\Delta x, y, t)-v(x, y, t)}{\phi_{3}}=\frac{R}{2}\left(v(x+\Delta x, y, t)-\frac{3}{4}\right)(1-v(x, y, t)) .
$$

In the same way as $u_{x x}$, we define $v_{x x}=\frac{v(x+\Delta x, y, t)-2 v(x, y, t)+v(x-\Delta x, y, t)}{\phi_{3} \phi_{4}}$, use (14), and we have

$$
\begin{aligned}
\frac{1}{R} v_{x x}= & \frac{v(x+\Delta x, y, t)-2 v(x, y, t)+v(x-\Delta x, y, t)}{R \phi_{3} \phi_{4}} \\
= & \frac{\frac{v(x+\Delta x, y, t)-v(x, y, t)}{\phi_{3}}-\frac{v(x, y, t)-v(x-\Delta x, y, t)}{\phi_{3}}}{R \phi_{4}} \\
= & \frac{\frac{R}{2}\left(v(x+\Delta x, y, t)-\frac{3}{4}\right)(1-v(x, y, t))-\frac{R}{2}\left(v(x, y, t)-\frac{3}{4}\right)(1-v(x-\Delta x, y, t))}{R \phi_{4}} \\
= & \frac{1}{2} \frac{\left(v(x+\Delta x, y, t)-\frac{3}{4}\right)(1-v(x, y, t))-\left(v(x, y, t)-\frac{3}{4}\right)(1-v(x-\Delta x, y, t))}{\phi_{4}} \\
= & \frac{v(x+\Delta x, y, t)-v(x, y, t)}{2 \phi_{4}}+\frac{3}{2} \frac{v(x, y, t)-v(x-\Delta x, y, t)}{4 \phi_{4}} \\
& -v(x, y, t) \frac{v(x+\Delta x, y, t)-v(x-\Delta x, y, t)}{2 \phi_{4}} .
\end{aligned}
$$

Equation (4) minus (4c) yields

$$
\begin{aligned}
\frac{1}{v(x, y, t)-\frac{3}{4}}-\frac{1}{v(x, y-\Delta y, t)-\frac{3}{4}} & =\left(1-e^{-\frac{R}{8} \Delta y}\right) 4 e^{\frac{R}{32}(4 y-4 x-t)} \\
& =\left(1-e^{-\frac{R}{8} \Delta y}\right)\left(\frac{1}{v(x, y, t)-\frac{3}{4}}-4\right) .
\end{aligned}
$$

Setting $\varphi_{4}=\left(1-e^{-\frac{R}{8} \Delta y}\right) /\left(\frac{R}{8}\right)$, we obtain

$$
\frac{v(x, y, t)-v(x, y-\Delta y, t)}{\varphi_{4}}=\frac{R}{2}\left(v(x, y-\Delta y, t)-\frac{3}{4}\right)(v(x, y, t)-1) .
$$

Define $v_{y y}=\frac{v(x, y+\Delta y, t)-2 v(x, y, t)+\nu(x, y-\Delta y, t)}{\varphi_{3} \varphi_{4}}$, on the basis of (17), we obtain the following result:

$$
\begin{aligned}
\frac{1}{R} v_{y y}= & \frac{v(x, y+\Delta y, t)-2 v(x, y, t)+v(x, y-\Delta y, t)}{R \varphi_{3} \varphi_{4}} \\
= & \frac{\frac{v(x+\Delta x, y, t)-v(x, y, t)}{\varphi_{4}}-\frac{v(x, y, t)-v(x-\Delta x, y, t)}{\phi_{4}}}{R \phi_{3}} \\
= & \frac{\frac{R}{2}\left(v(x, y, t)-\frac{3}{4}\right)(v(x, y+\Delta y, t)-1)-\frac{R}{2}\left(v(x, y-\Delta y, t)-\frac{3}{4}\right)(v(x, y, t)-1)}{R \varphi_{3}} \\
= & \frac{1}{2} \frac{\left(v(x, y, t)-\frac{3}{4}\right)(v(x, y+\Delta y, t)-1)-\left(v(x, y-\Delta y, t)-\frac{3}{4}\right)(v(x, y, t)-1)}{\varphi_{3}} \\
= & v(x, y, t) \frac{v(x, y+\Delta y, t)-v(x, y-\Delta y, t)}{2 \varphi_{3}}-\frac{v(x, y, t)-v(x, y-\Delta y, t)}{2 \varphi_{3}} \\
& -\frac{3}{2} \frac{v(x, y+\Delta y, t)-v(x, y, t)}{4 \varphi_{3}} .
\end{aligned}
$$


On the basis of (15) and (18), we have

$$
\begin{aligned}
\frac{1}{R} v_{x x}+\frac{1}{R} v_{y y}= & \frac{v(x+\Delta x, y, t)-v(x, y, t)}{2 \phi_{4}}+\frac{3}{2} \frac{v(x, y, t)-v(x-\Delta x, y, t)}{4 \phi_{4}} \\
& -v(x, y, t) \frac{v(x+\Delta x, y, t)-v(x-\Delta x, y, t)}{2 \phi_{4}} \\
& +v(x, y, t) \frac{v(x, y+\Delta y, t)-v(x, y-\Delta y, t)}{2 \varphi_{3}} \\
& -\frac{v(x, y, t)-v(x, y-\Delta y, t)}{2 \varphi_{3}}-\frac{3}{2} \frac{v(x, y+\Delta y, t)-v(x, y, t)}{4 \varphi_{3}} \\
= & \frac{v(x+\Delta x, y, t)-v(x, y, t)}{2 \phi_{4}}+\frac{3}{2} \frac{v(x, y, t)-v(x-\Delta x, y, t)}{4 \phi_{4}} \\
& -v(x, y, t) \frac{v(x+\Delta x, y, t)-v(x-\Delta x, y, t)}{2 \phi_{4}} \\
& +v(x, y, t) \frac{v(x, y+\Delta y, t)-v(x, y-\Delta y, t)}{2 \varphi_{3}} \\
& +\frac{v(x+\Delta x, y, t)-v(x, y, t)}{2 \varphi_{3}}+\frac{3}{2} \frac{v(x, y, t)-v(x-\Delta x, y, t)}{4 \varphi_{3}} .
\end{aligned}
$$

For $4 \Delta x=4 \Delta y=\Delta t$, so $\phi_{4}=\varphi_{3}$, set $\psi_{4}=\left(e^{\frac{R}{32} \Delta t}-1\right) /\left(\frac{R}{32}\right)$, we have

$$
\begin{aligned}
\frac{1}{R} v_{x x}+\frac{1}{R} v_{y y}= & \frac{3}{2} \frac{v(x+\Delta x, y, t)-v(x, y, t)}{2 \phi_{4}}+\frac{3}{2} \frac{v(x, y, t)-v(x-\Delta x, y, t)}{2 \phi_{4}} \\
& -v(x, y, t) \frac{v(x+\Delta x, y, t)-v(x-\Delta x, y, t)}{2 \phi_{4}} \\
& +v(x, y, t) \frac{v(x, y+\Delta y, t)-v(x, y-\Delta y, t)}{2 \varphi_{3}} \\
& +\frac{1}{2} \frac{v(x+\Delta x, y, t)-v(x, y, t)}{2 \phi_{4}} \\
= & \left.\frac{3}{2}-v(x, y, t)\right) \frac{v(x+\Delta x, y, t)-v(x-\Delta x, y, t)}{2 \phi_{4}} \\
& +\frac{v(x+\Delta x, y, t)-v(x, y, t)}{4 \phi_{4}}+v(x, y, t) \frac{v(x, y+\Delta y, t)-v(x, y-\Delta y, t)}{2 \varphi_{3}} \\
= & \left(\frac{3}{2}-v(x, y, t)\right) \frac{v(x+\Delta x, y, t)-v(x-\Delta x, y, t)}{2 \phi_{4}} \\
& +\frac{v(x, y, t+\Delta t)-v(x, y, t)}{\psi_{4}} \\
& +v(x, y, t) \frac{v(x, y+\Delta y, t)-v(x, y-\Delta y, t)}{2 \varphi_{3}} \\
& +v_{t}+v v_{y} .
\end{aligned}
$$

Equation (20) is the explicit discrete format of the second equation in (1).

\subsection{Implicit exact-difference scheme}

We will give the implicit format of (1) in the following analysis. 


\subsubsection{Discrete scheme for the first equation}

Furthermore, if we use (3) minus (3b)

$$
\begin{aligned}
\frac{1}{\frac{3}{4}-u(x, y, t)}-\frac{1}{\frac{3}{4}-u(x-\Delta x, y, t)} & =\left(1-e^{\frac{R}{8} \Delta x}\right) 4 e^{\frac{R}{32}(4 y-4 x-t)} \\
& =\left(1-e^{\frac{R}{8} \Delta x}\right)\left(\frac{1}{\frac{3}{4}-u(x, y, t)}-4\right) .
\end{aligned}
$$

According to (21), we assume $\phi_{2}=\left(e^{\frac{R}{8} \Delta x}-1\right) /\left(\frac{R}{8}\right)$. We obtain another result:

$$
\frac{u(x, y, t)-u(x-\Delta x, y, t)}{\phi_{2}}=\frac{R}{4}\left(\frac{3}{4}-u(x-\Delta x, y, t)\right)(1-2 u(x, y, t)) .
$$

If we substitute (22) into $u_{x x}$, we will get the following result:

$$
\begin{aligned}
& \frac{1}{R} u_{x x}=\frac{u(x+\Delta x, y, t)-2 u(x, y, t)+u(x-\Delta x, y, t)}{R \phi_{1} \phi_{2}} \\
& =\frac{\frac{u(x+\Delta x, y, t)-u(x, y, t)}{\phi_{2}}-\frac{u(x, y, t)-u(x-\Delta x, y, t)}{\phi_{2}}}{R \phi_{1}} \\
& =\frac{\frac{R}{4}\left(\frac{3}{4}-u(x, y, t)\right)(1-2 u(x+\Delta x, y, t))-\frac{R}{4}\left(\frac{3}{4}-u(x-\Delta x, y, t)\right)(1-2 u(x, y, t))}{R \phi_{1}} \\
& =\frac{1}{4} \frac{\left(\frac{3}{4}-u(x, y, t)\right)(1-2 u(x+\Delta x, y, t))-\left(\frac{3}{4}-u(x-\Delta x, y, t)\right)(1-2 u(x, y, t))}{\phi_{1}} \\
& =\frac{u(x-\Delta x, y, t)-u(x, y, t)}{4 \phi_{1}}-\frac{3}{2} \frac{u(x+\Delta x, y, t)-u(x, y, t)}{4 \phi_{1}} \\
& +u(x, y, t) \frac{u(x+\Delta x, y, t)-u(x-\Delta x, y, t)}{2 \phi_{1}} .
\end{aligned}
$$

Equation (3d) minus (3) yields

$$
\begin{aligned}
\frac{1}{\frac{3}{4}-u(x, y+\Delta y, t)}-\frac{1}{\frac{3}{4}-u(x, y, t)} & =\left(e^{\frac{R}{8} \Delta y}-1\right) 4 e^{\frac{R}{32}(4 y-4 x-t)} \\
& =\left(e^{\frac{R}{8} \Delta y}-1\right)\left(\frac{1}{\frac{3}{4}-u(x, y, t)}-4\right) .
\end{aligned}
$$

By setting $\varphi_{1}=\left(e^{\frac{R}{8} \Delta y}-1\right) /\left(\frac{R}{8}\right)$, we have

$$
\frac{u(x, y+\Delta y, t)-u(x, y, t)}{\varphi_{1}}=\frac{R}{4}\left(\frac{3}{4}-u(x, y+\Delta y, t)\right)(2 u(x, y, t)-1) .
$$

Referring to (25), we get the following equation:

$$
\begin{aligned}
\frac{1}{R} u_{y y} & =\frac{u(x, y+\Delta y, t)-2 u(x, y, t)+u(x, y-\Delta y, t)}{R \varphi_{1} \varphi_{2}} \\
& =\frac{\frac{u(x, y+\Delta y, t)-u(x, y, t)}{\varphi_{1}}-\frac{u(x, y, t)-u(x, y-\Delta y, t)}{\varphi_{1}}}{R \varphi_{2}}
\end{aligned}
$$




$$
\begin{aligned}
= & \frac{\frac{R}{4}\left(\frac{3}{4}-u(x, y+\Delta y, t)\right)(2 u(x, y, t)-1)-\frac{R}{4}\left(\frac{3}{4}-u(x, y, t)\right)(2 u(x, y-\Delta y, t)-1)}{R \varphi_{2}} \\
= & \frac{1}{4} \frac{\left(\frac{3}{4}-u(x, y+\Delta y, t)\right)(2 u(x, y, t)-1)-\left(\frac{3}{4}-u(x, y, t)\right)(2 u(x, y-\Delta y, t)-1)}{\varphi_{2}} \\
= & \frac{u(x, y+\Delta y, t)-u(x, y, t)}{4 \varphi_{2}}+\frac{3}{2} \frac{u(x, y, t)-u(x, y-\Delta y, t)}{4 \varphi_{2}} \\
& -u(x, y, t) \frac{u(x, y+\Delta y, t)-u(x, y-\Delta y, t)}{2 \varphi_{2}} .
\end{aligned}
$$

And on the basis of (23) and (26), we have

$$
\begin{aligned}
\frac{1}{R} u_{x x}+\frac{1}{R} u_{y y}= & \frac{u(x-\Delta x, y, t)-u(x, y, t)}{4 \phi_{1}}-\frac{3}{2} \frac{u(x+\Delta x, y, t)-u(x, y, t)}{4 \phi_{1}} \\
& +u(x, y, t) \frac{u(x+\Delta x, y, t)-u(x-\Delta x, y, t)}{2 \phi_{1}} \\
& +\frac{u(x, y+\Delta y, t)-u(x, y, t)}{4 \varphi_{2}}+\frac{3}{2} \frac{u(x, y, t)-u(x, y-\Delta y, t)}{4 \varphi_{2}} \\
& -u(x, y, t) \frac{u(x, y+\Delta y, t)-u(x, y-\Delta y, t)}{2 \varphi_{2}} \\
= & \frac{u(x, y+\Delta y, t)-u(x, y, t)}{4 \phi_{1}}+\frac{3}{2} \frac{u(x, y, t)-u(x, y-\Delta y, t)}{4 \phi_{1}} \\
& +u(x, y, t) \frac{u(x+\Delta x, y, t)-u(x-\Delta x, y, t)}{2 \phi_{1}} \\
& +\frac{u(x, y+\Delta y, t)-u(x, y, t)}{4 \varphi_{2}}+\frac{3}{2} \frac{u(x, y, t)-u(x, y-\Delta y, t)}{4 \varphi_{2}} \\
& +u \frac{u(x, y+\Delta y, t)-u(x, y-\Delta y, t)}{4 \varphi_{2}}-\frac{1}{2} \frac{u(x, y+\Delta y, t)-u(x, y, t)}{4 \varphi_{2}} \\
& -\frac{3}{2} \frac{u(x, y+\Delta y, t)-u(x, y-\Delta y, t)}{4 \phi_{1}}-\frac{1}{2} \frac{u(x, y+\Delta y, t)-u(x, y, t)}{4 \phi_{1}} \\
& +u(x, y, t) \frac{u(x, y+\Delta y, t)-u(x, y-\Delta y, t)}{2 \varphi_{2}} \\
& +u(x, y-\Delta y, t) \\
& \\
& +u(x, y, t)-u(x, y, t) \\
& \\
& \\
&
\end{aligned}
$$

Assume $\psi_{2}=\left(1-e^{-\frac{R}{32} \Delta t}\right) /\left(\frac{R}{32}\right)$. We already know $4 \Delta x=4 \Delta y=\Delta t$, so $\phi_{1}=\varphi_{2}$, and we obtain

$$
\begin{aligned}
\frac{1}{R} u_{x x}+\frac{1}{R} u_{y y}= & u(x, y, t) \frac{u(x+\Delta x, y, t)-u(x-\Delta x, y, t)}{2 \phi_{1}} \\
& +\frac{3}{2} \frac{u(x, y+\Delta y, t)-u(x, y-\Delta y, t)}{2 \varphi_{2}}-\frac{u(x, y+\Delta y, t)-u(x, y, t)}{4 \varphi_{2}} \\
& -u(x, y, t) \frac{u(x, y+\Delta y, t)-u(x, y-\Delta y, t)}{2 \varphi_{2}}
\end{aligned}
$$




$$
\begin{aligned}
= & u(x, y, t) \frac{u(x+\Delta x, y, t)-u(x-\Delta x, y, t)}{2 \phi_{1}}-\frac{u(x, y+\Delta y, t)-u(x, y, t)}{4 \varphi_{2}} \\
& +\left(\frac{3}{2}+u(x, y, t)\right) \frac{u(x, y+\Delta y, t)-u(x, y-\Delta y, t)}{2 \varphi_{2}} \\
= & u(x, y, t) \frac{u(x+\Delta x, y, t)-u(x-\Delta x, y, t)}{2 \phi_{1}}+\frac{u(x, y, t)-u(x, y, t-\Delta t)}{\psi_{2}} \\
& +v(x, y, t) \frac{u(x, y+\Delta y, t)-u(x, y-\Delta y, t)}{2 \varphi_{2}} \\
\doteq & u u_{x}+u_{t}+v u_{y} .
\end{aligned}
$$

Equation (28) is the implicit discrete format for the first equation of (1). Now, we will give the implicit scheme for the second equation of (1).

\subsubsection{Discrete scheme for the second equation}

If we use (4) minus (4b), we immediately get

$$
\begin{aligned}
\frac{1}{v(x, y, t)-\frac{3}{4}}-\frac{1}{v(x-\Delta x, y, t)-\frac{3}{4}} & =\left(1-e^{\frac{R}{8} \Delta x}\right) 4 e^{\frac{R}{32}(4 y-4 x-t)} \\
& =\left(1-e^{\frac{R}{8} \Delta x}\right)\left(\frac{1}{v(x, y, t)-\frac{3}{4}}-4\right) .
\end{aligned}
$$

Set $\phi_{4}=\left(e^{\frac{R}{8} \Delta x}-1\right) /\left(\frac{R}{8}\right)$, then we have

$$
\frac{v(x, y, t)-v(x-\Delta x, y, t)}{\phi_{4}}=\frac{R}{2}\left(v(x-\Delta x, y, t)-\frac{3}{4}\right)(1-v(x, y, t)) .
$$

Using (30), we obtain

$$
\begin{aligned}
\frac{1}{R} v_{x x}= & \frac{v(x+\Delta x, y, t)-2 v(x, y, t)+v(x-\Delta x, y, t)}{R \phi_{3} \phi_{4}} \\
= & \frac{\frac{v(x+\Delta x, y, t)-v(x, y, t)}{\phi_{4}}-\frac{v(x, y, t)-v(x-\Delta x, y, t)}{\phi_{4}}}{R \phi_{3}} \\
= & \frac{\frac{R}{2}\left(v(x, y, t)-\frac{3}{4}\right)(1-v(x+\Delta x, y, t))-\frac{R}{2}\left(v(x-\Delta x, y, t)-\frac{3}{4}\right)(1-v(x, y, t))}{R \phi_{3}} \\
= & \frac{1}{2} \frac{\left(v(x, y, t)-\frac{3}{4}\right)(1-v(x+\Delta x, y, t))-\left(v(x-\Delta x, y, t)-\frac{3}{4}\right)(1-v(x, y, t))}{\phi_{3}} \\
= & \frac{v(x, y, t)-v(x-\Delta x, y, t)}{2 \phi_{3}}+\frac{3}{2} \frac{v(x+\Delta x, y, t)-v(x, y, t)}{4 \phi_{3}} \\
& -v(x, y, t) \frac{v(x+\Delta x, y, t)-v(x-\Delta x, y, t)}{2 \phi_{3}} .
\end{aligned}
$$

Using (4d) minus (4), we immediately get

$$
\begin{aligned}
\frac{1}{v(x, y+\Delta y, t)-\frac{3}{4}}-\frac{1}{v(x, y, t)-\frac{3}{4}} & =\left(e^{\frac{R}{8} \Delta y}-1\right) 4 e^{\frac{R}{32}(4 y-4 x-t)} \\
& =\left(e^{\frac{R}{8} \Delta y}-1\right)\left(\frac{1}{v(x, y, t)-\frac{3}{4}}-4\right) .
\end{aligned}
$$


Setting $\varphi_{3}=\left(e^{\frac{R}{8} \Delta y}-1\right) /\left(\frac{R}{8}\right)$, we have

$$
\frac{v(x, y+\Delta y, t)-v(x, y, t)}{\varphi_{3}}=\frac{R}{2}\left(v(x, y+\Delta y, t)-\frac{3}{4}\right)(v(x, y, t)-1) .
$$

According to (33), we have

$$
\begin{aligned}
\frac{1}{R} v_{y y}= & \frac{v(x, y+\Delta y, t)-2 v(x, y, t)+v(x, y-\Delta y, t)}{R \varphi_{3} \varphi_{4}} \\
= & \frac{\frac{v(x, y+\Delta y, t)-v(x, y, t)}{\varphi_{3}}-\frac{v(x, y, t)-v(x, y-\Delta y, t)}{\varphi_{3}}}{R \varphi_{4}} \\
= & \frac{\frac{R}{2}\left(v(x, y+\Delta y, t)-\frac{3}{4}\right)(v(x, y, t)-1)-\frac{R}{2}\left(v(x, y, t)-\frac{3}{4}\right)(v(x, y-\Delta y, t)-1)}{R \varphi_{4}} \\
= & \frac{1}{2} \frac{\left(v(x, y+\Delta y, t)-\frac{3}{4}\right)(v(x, y, t)-1)-\left(v(x, y, t)-\frac{3}{4}\right)(v(x, y-\Delta y, t)-1)}{\varphi_{4}} \\
= & v(x, y, t) \frac{v(x, y+\Delta y, t)-v(x, y-\Delta y, t)}{2 \varphi_{4}}-\frac{v(x, y+\Delta y, t)-v(x, y, t)}{2 \varphi_{4}} \\
& -\frac{3}{2} \frac{v(x, y, t)-v(x, y-\Delta y, t)}{4 \varphi_{4}} .
\end{aligned}
$$

According to (31) and (34), we obtain

$$
\begin{aligned}
\frac{1}{R} v_{x x}+\frac{1}{R} v_{y y}= & \frac{v(x, y, t)-v(x-\Delta x, y, t)}{2 \phi_{3}}+\frac{3}{2} \frac{v(x+\Delta x, y, t)-v(x, y, t)}{4 \phi_{3}} \\
& -v(x, y, t) \frac{v(x+\Delta x, y, t)-v(x-\Delta x, y, t)}{2 \phi_{3}} \\
& +v(x, y, t) \frac{v(x, y+\Delta y, t)-v(x, y-\Delta y, t)}{2 \varphi_{4}} \\
& -\frac{v(x, y+\Delta y, t)-v(x, y, t)}{2 \varphi_{4}}-\frac{3}{2} \frac{v(x, y, t)-v(x, y-\Delta y, t)}{4 \varphi_{4}} \\
= & \frac{v(x, y, t)-v(x-\Delta x, y, t)}{2 \phi_{3}}+\frac{3}{2} \frac{v(x+\Delta x, y, t)-v(x, y, t)}{4 \phi_{3}} \\
& -v(x, y, t) \frac{v(x+\Delta x, y, t)-v(x-\Delta x, y, t)}{2 \phi_{3}} \\
& +v(x, y, t) \frac{v(x, y+\Delta y, t)-v(x, y-\Delta y, t)}{2 \varphi_{4}} \\
& +\frac{v(x, y, t)-v(x-\Delta x, y, t)}{2 \varphi_{4}}+\frac{3}{2} \frac{v(x+\Delta x, y, t)-v(x, y, t)}{4 \varphi_{4}} .
\end{aligned}
$$

Because $4 \Delta x=4 \Delta y=\Delta t$, so $\phi_{3}=\varphi_{4}$, setting $\psi_{3}=\left(1-e^{-\frac{R}{32} \Delta t}\right) /\left(\frac{R}{32}\right)$, we have

$$
\begin{aligned}
\frac{1}{R} v_{x x}+\frac{1}{R} v_{y y}= & \frac{3}{2} \frac{v(x, y, t)-v(x-\Delta x, y, t)}{2 \phi_{3}}+\frac{3}{2} \frac{v(x+\Delta x, y, t)-v(x, y, t)}{2 \phi_{3}} \\
& -v(x, y, t) \frac{v(x+\Delta x, y, t)-v(x-\Delta x, y, t)}{2 \phi_{3}} \\
& +v(x, y, t) \frac{v(x, y+\Delta y, t)-v(x, y-\Delta y, t)}{2 \varphi_{4}}+\frac{1}{2} \frac{v(x, y, t)-v(x-\Delta x, y, t)}{2 \phi_{3}}
\end{aligned}
$$




$$
\begin{aligned}
= & \left(\frac{3}{2}-v(x, y, t)\right) \frac{v(x+\Delta x, y, t)-v(x-\Delta x, y, t)}{2 \phi_{3}} \\
& +\frac{v(x, y, t)-v(x-\Delta x, y, t)}{4 \phi_{3}}+v(x, y, t) \frac{v(x, y+\Delta y, t)-v(x, y-\Delta y, t)}{2 \varphi_{4}} \\
= & \left(\frac{3}{2}-v(x, y, t)\right) \frac{v(x+\Delta x, y, t)-v(x-\Delta x, y, t)}{2 \phi_{3}} \\
& +\frac{v(x, y, t)-v(x, y, t-\Delta t)}{\psi_{3}}+v(x, y, t) \frac{v(x, y+\Delta y, t)-v(x, y-\Delta y, t)}{2 \varphi_{4}} \\
\doteq & u v_{x}+v_{t}+v v_{y} .
\end{aligned}
$$

Now, we can find that (36) is the implicit discrete format of the second equation in (1). In the next step, we will give the exact finite-difference schemes.

\subsection{The exact finite schemes for coupled Burgers equation}

We denote the discrete approximation of $u(x, y, t)$ and $v(x, y, t)$ at the mesh point $(i \Delta x ; j \Delta y$; $n \Delta t)$ by $U_{i, j}^{n}$ and $V_{i, j}^{n}$, respectively, $i=0,1,2, \ldots, N_{x} ; j=0,1,2, \ldots, N_{y} ; n=0,1,2, \ldots$, where $\Delta x=\frac{1}{N_{x}}$ is the mesh size in $x$ direction and $\Delta y=\frac{1}{N_{y}}$ is the mesh size in $y$ direction, and $\tau$ represents the increment in time. Set $\Delta x=\Delta y=h$, we write $U_{i, j}^{n}$ as

$$
\left\{\begin{array}{l}
U_{i, j}^{n}=u\left(x_{i}, y_{j}, t_{n}\right)=\frac{3}{4}-\frac{1}{4\left(1+e^{\frac{R}{32}\left(4 y_{j}-4 x_{i}-t_{n}\right)}\right)}, \\
V_{i, j}^{n}=v\left(x_{i}, y_{j}, t_{n}\right)=\frac{3}{4}+\frac{1}{4\left(1+e^{\frac{R}{32}\left(4 y_{j}-4 x_{i}-t_{n}\right)}\right)} .
\end{array}\right.
$$

For $4 \Delta x=4 \Delta y=\Delta t, \psi_{1}=\left(e^{\frac{R}{22} \Delta t}-1\right) /\left(\frac{R}{32}\right)=\psi_{4}, \phi_{2}=\varphi_{1}, \phi_{4}=\varphi_{3}$, based on (12) and (20), we have the explicit difference scheme

$$
\left\{\begin{array}{l}
\frac{U_{i+1, j}^{n}-2 U_{i, j}^{n}+U_{i-1, j}^{n}}{R \phi_{1} \phi_{2}}+\frac{U_{i, j+1}^{n}-2 U_{i, j}^{n}+U_{i, j-1}^{n}}{R \varphi_{1} \varphi_{2}} \\
=U_{i, j}^{n} \frac{U_{i+1, j}^{n}-U_{i-1, j}^{n}}{2 \phi_{2}}+\frac{U_{i, j}^{n+1}-U_{i, j}^{n}}{\psi_{1}^{n}}+V_{i, j}^{n} \frac{U_{i, j+1}^{n}-U_{i, j-1}^{n}}{2 \varphi_{1}}, \\
\frac{V_{i+1, j}^{n}-2 V_{i, j}^{n}+V_{i-1, j}^{n}}{R \phi_{3} \phi_{4}}+\frac{V_{i, j+1}^{n}-2 V_{i, j}^{n}+v_{i, j-1}^{n}}{R \varphi_{3} \varphi_{4}} \\
=U_{i, j}^{n} \frac{V_{i+1, j}^{n}-V_{i-1, j}^{n}}{2 \phi_{4}}+\frac{V_{i, j}^{n+1}-V_{i, j}^{n}}{\psi_{4}}+V_{i, j}^{n} \frac{V_{i, j+1}^{n}-V_{i, j-1}^{n}}{2 \varphi_{3}} .
\end{array}\right.
$$

For $4 \Delta x=4 \Delta y=\Delta t, \psi_{2}=\left(1-e^{-\frac{R}{32} \Delta t}\right) /\left(\frac{R}{32}\right)=\psi_{3}, \phi_{1}=\varphi_{2}, \phi_{3}=\varphi_{4}$, according to (28) and (36), we can obtain an implicit difference scheme

$$
\left\{\begin{array}{l}
\frac{U_{i+1, j}^{n}-2 U_{i, j}^{n}+U_{i-1, j}^{n}}{R \phi_{1} \phi_{2}}+\frac{U_{i, j+1}^{n}-2 U_{i, j}^{n}+U_{i, j-1}^{n}}{R \varphi_{1} \varphi_{2}} \\
=U_{i, j}^{n} U_{i+1, j}^{n}-U_{i-1, j}^{n}+\frac{U_{i, j}^{n}-U_{i, j}^{n-1}}{\psi_{2}}+V_{i, j}^{n} \frac{U_{i, j+1}^{n}-U_{i, j-1}^{n}}{2 \varphi_{2}}, \\
\frac{V_{i+1, j}^{n}-2 V_{i, j}^{n}+V_{i-1, j}^{n}}{R \phi_{3} \phi_{4}}+\frac{V_{i, j+1}^{n}-2 V_{i, j}^{n}+V_{i, j-1}^{n}}{R \varphi_{3} \varphi_{4}} \\
=U_{i, j}^{n} \frac{V_{i+1, j}^{n}-V_{i-1, j}^{n}}{2 \phi_{3}}+\frac{V_{i, j}^{n}-V_{i, j}^{n-1}}{\psi_{3}}+V_{i, j}^{n} \frac{V_{i, j+1}^{n}-V_{i, j-1}^{n}}{2 \varphi_{4}} .
\end{array}\right.
$$

Theorem 2.1 The difference schemes (38) and (39) are the exact explicit and implicit difference schemes for (1), where $\phi_{i}, \varphi_{i}, \psi_{i}$ are the expressions in Sections 2.1 and 2.2.

Proof We can see the analysis from Section 2.1 and Section 2.2 that the two schemes are explicit and implicit exact finite schemes. 


\section{Nonstandard finite-difference scheme for coupled Burgers equation}

We gave the exact finite-difference schemes in Section 2. In this section, we will give two nonstandard finite-difference schemes using two different ways.

\subsection{The first nonstandard finite-difference scheme}

First, based on the exact schemes, we can see that the exact numerical schemes (38) and (39) of (1) have some conditions in steps. If we select the exact schemes, but a different stepsize, the exact scheme will become a nonstandard finite-difference scheme. In order to facilitate the calculation, we choose this explicit form in our paper:

$$
\left\{\begin{array}{l}
\frac{U_{i+1, j}^{n}-2 U_{i, j}^{n}+U_{i-1, j}^{n}}{R \phi_{1} \phi_{2}}+\frac{U_{i, j+1}^{n}-2 U_{i, j}^{n}+U_{i, j-1}^{n}}{R \varphi_{1} \varphi_{2}} \\
=U_{i, j}^{n} \frac{U_{i+1, j}^{n}-U_{i-1, j}^{n}}{2 \phi_{2}}+\frac{U_{i, j}^{n+1}-U_{i, j}^{n}}{\psi_{1}}+V_{i, j}^{n} \frac{U_{i, j+1}^{n}-U_{i, j-1}^{n}}{2 \varphi_{1}}, \\
\frac{V_{i+1, j}^{n}-2 V_{i, j}^{n}+V_{i-1, j}^{n}}{R \phi_{3} \phi_{4}}+\frac{V_{i, j+1}^{n}-2 V_{i, j}^{n}+v_{i, j-1}^{n}}{R \varphi_{3} \varphi_{4}} \\
=U_{i, j}^{n} \frac{V_{i+1, j}^{n}-V_{i-1, j}^{n}}{2 \phi_{4}}+\frac{V_{i, j}^{n+1}-V_{i, j}^{n}}{\psi_{4}}+V_{i, j}^{n} \frac{V_{i, j+1}^{n}-V_{i, j-1}^{n}}{2 \varphi_{3}}
\end{array}\right.
$$

where the step function is of the same form as in Section 2.

\subsection{The second nonstandard finite-difference scheme}

We already noticed that the stepsize for exact schemes in Section 2 must satisfy some fixed conditions. In order to relax the conditions for stepsize, we would like to use a different way of studying [9-14] to construct nonstandard finite-difference schemes for (1). Referring to Zhang et al. [14], we set the exact scheme

$$
\left\{\begin{array}{c}
\frac{U_{i, j}^{n+1}-U_{i, j}^{n}}{\phi(\Delta t)}+U_{i, j}^{n+1} \frac{U_{i+1, j}^{n}-U_{i-1, j}^{n}}{2 \psi(\Delta x)}+V_{i, j}^{n} \frac{U_{i, j+1}^{n}-U_{i, j-1}^{n}}{2 \psi(\Delta y)} \\
=\frac{U_{i+1, j}^{n}-2 U_{i, j}^{n}+U_{i-1, j}^{n}}{R \psi(\Delta x)^{2}}+\frac{U_{i, j+1}^{n}-2 U_{i, j}^{n}+U_{i, j-1}^{n}}{R \psi(\Delta y)^{2}}, \\
\frac{V_{i, j}^{n+1}-V_{i, j}^{n}}{\varphi(\Delta t)}+U_{i, j}^{n} \frac{V_{i+1, j}^{n}-V_{i-1, j}^{n}}{2 \psi(\Delta x)}+V_{i, j}^{n+1} \frac{V_{i, j+1}^{n}-V_{i, j-1}^{n}}{2 \psi(\Delta y)} \\
=\frac{V_{i+1, j}^{n}-2 V_{i, j}^{n}+V_{i-1, j}^{n}}{R \psi(\Delta x)^{2}}+\frac{V_{i, j+1}^{n}-2 V_{i, j}^{n}+V_{i, j-1}^{n}}{R \psi(\Delta y)^{2}} .
\end{array}\right.
$$

Using the way of Zhang et al. [14] and the notation (37), setting $\Delta x=\Delta y=h$, we obtain

$$
\begin{aligned}
\phi(\Delta t)= & \frac{U_{i, j}^{n+1}-U_{i, j}^{n}}{\frac{U_{i+1, j}^{n}-2 U_{i, j}^{n}+U_{i-1, j}^{n}}{R \psi^{2}(h)}+\frac{U_{i, j+1}^{n}-2 U_{i, j}^{n}+U_{i, j-1}^{n}}{R \psi^{2}(h)}-U_{i, j}^{n+1} \frac{U_{i+1, j}^{n}-U_{i-1, j}^{n}}{2 \psi(h)}-V_{i, j}^{n} \frac{U_{i, j+1}^{n}-U_{i, j-1}^{n}}{2 \psi(h)}} \\
= & 2 R \psi^{2}(h)\left(U_{i, j}^{n+1}-U_{i, j}^{n}\right) /\left(2\left(U_{i+1, j}^{n}-2 U_{i, j}^{n}+U_{i-1, j}^{n}\right)+2\left(U_{i, j+1}^{n}-2 U_{i, j}^{n}+U_{i, j-1}^{n}\right)\right. \\
& \left.-R \psi(h) U_{i, j}^{n+1}\left(U_{i+1, j}^{n}-U_{i-1, j}^{n}\right)-R \psi(h) V_{i, j}^{n}\left(U_{i, j+1}^{n}-U_{i, j-1}^{n}\right)\right), \\
\varphi(\Delta t)= & \frac{V_{i, j}^{n+1}-V_{i, j}^{n}}{V_{i+1, j}^{n}-2 V_{i, j}^{n}+V_{i-1, j}^{n}+\frac{V_{i, j+1}^{n}-2 V_{i, j}^{n}+V_{i, j-1}^{n}}{R \psi^{2}(h)}-U_{i, j}^{n} \frac{V_{i+1, j}^{n}-V_{i-1, j}^{n}}{2 \psi(h)}-V_{i, j}^{n+1} \frac{V_{i, j+1}^{n}-V_{i, j-1}^{n}}{2 \psi(h)}} \\
= & 2 R \psi^{2}(h)\left(V_{i, j}^{n+1}-V_{i, j}^{n}\right) /\left(2\left(V_{i+1, j}^{n}-2 V_{i, j}^{n}+V_{i-1, j}^{n}\right)+2\left(V_{i, j+1}^{n}-2 V_{i, j}^{n}+V_{i, j-1}^{n}\right)\right. \\
& \left.-R \psi(h) U_{i, j}^{n}\left(V_{i+1, j}^{n}-V_{i-1, j}^{n}\right)-R \psi(h) V_{i, j}^{n+1}\left(V_{i, j+1}^{n}-V_{i, j-1}^{n}\right)\right) .
\end{aligned}
$$


If we choose $\psi(h)=\frac{8\left(e^{\frac{R}{8} h}-1\right)}{R}$, we have

$$
\left\{\begin{array}{l}
\phi(\Delta t)=\frac{64\left(1-e^{-\frac{R}{32} \Delta t}\right)\left(1+e^{-\frac{R}{8} h} s\right)\left(1+e^{\frac{R}{8} h} s\right)}{R\left(2\left(1+e^{-\frac{R}{32} \Delta t} s\right)(s-1) e^{-\frac{R}{8} h}+\left(1+e^{-\frac{R}{8} h}\right)\left(e^{-\frac{R}{32} \Delta t} s+s+2\right)\right)}, \\
\varphi(\Delta t)=\frac{64\left(1-e^{-\frac{R}{32} \Delta t}\right)\left(1+e^{-\frac{R}{8} h} s\right)\left(1+e^{\frac{R}{8} h} s\right)}{R\left(2\left(1+e^{-\frac{R}{32} \Delta t} s\right)(s-1) e^{-\frac{R}{8} h}+\left(1+e^{-\frac{R}{8} h}\right)\left(e^{-\frac{R}{32} \Delta t} s+s+2\right)\right)}
\end{array}\right.
$$

where $s=s_{i, j}^{n}=e^{\frac{R}{32}\left(4 y_{j}-4 x_{i}-t_{n}\right)}$. We use $s$ instead of $s_{i, j}^{n}$ for convenience.

If $\psi$ satisfy $\psi(\Delta x)=\psi(\Delta y)=\psi(h)=h+O\left(h^{2}\right)$ when $h \rightarrow 0, \Delta t \rightarrow 0$, we can easily obtain $\phi(\Delta t) \rightarrow 32\left(1-e^{-\frac{\Delta t}{32}}\right) / R$ and $\varphi(\Delta t) \rightarrow 32\left(1-e^{-\frac{\Delta t}{32}}\right) / R$, with $\phi(\Delta t)=\varphi(\Delta t)=\Delta t+O\left(\Delta t^{2}\right)$. So when $\Delta x, \Delta y$ and $\Delta t$ approach zero, we obtain another nonstandard finite-difference scheme for the Burgers equation as follows:

$$
\left\{\begin{array}{l}
\frac{U_{i, j}^{n+1}-U_{i, j}^{n}}{\phi(\Delta t)}+U_{i, j}^{n+1} \frac{U_{i+1, j}^{n}-U_{i-1, j}^{n}}{2 \psi(\Delta x)}+V_{i, j}^{n} \frac{U_{i, j+1}^{n}-U_{i, j-1}^{n}}{2 \psi(\Delta y)} \\
=\frac{U_{i+1, j}^{n}-2 U_{i, j}^{n}+U_{i-1, j}^{n}}{R \psi(\Delta x)^{2}}+\frac{U_{i, j+1}^{n}-2 U_{i, j}^{n}+U_{i, j-1}^{n}}{R \psi(\Delta y)^{2}}, \\
\frac{V_{i, j}^{n+1}-V_{i, j}^{n}}{\varphi(\Delta t}+U_{i, j}^{n} \frac{V_{i+1, j}^{n}-V_{i-1, j}^{n}}{2 \psi(\Delta x)}+V_{i, j}^{n+1} \frac{V_{i, j+1}^{n}-V_{i, j-1}^{n}}{2 \psi(\Delta y)} \\
\quad=\frac{V_{i+1, j}^{n}-2 V_{i, j}^{n}+V_{i-1, j}^{n}}{R \psi(\Delta x)^{2}}+\frac{V_{i, j+1}^{n}-2 V_{i, j}^{n}+V_{i, j-1}^{n}}{R \psi(\Delta y)^{2}}, \\
\psi(\Delta x)=\psi(\Delta y)=8\left(e^{\frac{R}{8} h}-1\right) / R, \\
\phi(\Delta t)=\varphi(\Delta t)=32\left(1-e^{-\frac{\Delta t}{32}}\right) / R .
\end{array}\right.
$$

From the above analysis, we can see that our schemes are explicit. We will illustrate the efficiency and accuracy of these two nonstandard finite-difference schemes in the numerical simulation.

\section{Numerical experiments}

To illustrate the efficiency and accuracy of our nonstandard finite-difference schemes, we simulate the following initial-boundary problem:

$$
\left\{\begin{array}{l}
u_{t}+u u_{x}+v u_{y}=\frac{1}{R}\left(u_{x x}+u_{y y}\right), \\
v_{t}+u v_{x}+v v_{y}=\frac{1}{R}\left(v_{x x}+v_{y y}\right)
\end{array}\right.
$$

with the initial conditions

$$
\begin{cases}u(x, y, 0)=f(x, y), & (x, y) \in \Omega, \\ v(x, y, 0)=g(x, y), & (x, y) \in \Omega\end{cases}
$$

and the boundary conditions

$$
\begin{cases}u(x, y, t)=f_{1}(x, y, t), & x, y \in \partial \Omega, t>0, \\ v(x, y, t)=g_{1}(x, y, t), & x, y \in \partial \Omega, t>0 .\end{cases}
$$

For the nonstandard finite-difference scheme (40), $U_{i, j}^{n+1}$ and $V_{i, j}^{n+1}$ can be written as

$$
\left\{\begin{aligned}
U_{i, j}^{n+1}= & \psi_{1}\left[\frac{U_{i+1, j}^{n}-2 U_{i, j}^{n}+U_{i-1, j}^{n}}{R \phi_{1} \phi_{2}}+\frac{U_{i, j+1}^{n}-2 U_{i, j}^{n}+U_{i, j-1}^{n}}{R \varphi_{1} \varphi_{2}}-V_{i, j}^{n} \frac{U_{i, j+1}^{n}-U_{i, j-1}^{n}}{2 \varphi_{1}}\right. \\
& \left.-U_{i, j}^{n} \frac{U_{i+1, j}^{n}-U_{i-1, j}^{n}}{2 \phi_{2}}\right]+U_{i, j}^{n}, \\
V_{i, j}^{n+1}= & \psi_{4}\left[\frac{V_{i+1, j}^{n}-2 V_{i, j}^{n}+V_{i-1, j}^{n}}{R \phi_{3} \phi_{4}}+\frac{V_{i, j+1}^{n}-2 V_{i, j}^{n}+v_{i, j-1}^{n}}{R \varphi_{3} \varphi_{4}}-U_{i, j}^{n} \frac{V_{i+1, j}^{n}-V_{i-1, j}^{n}}{2 \phi_{4}}\right. \\
& \left.-V_{i, j}^{n} \frac{V_{i, j+1}^{n}-V_{i, j-1}^{n}}{2 \varphi_{3}}\right]+V_{i, j}^{n} .
\end{aligned}\right.
$$


For the nonstandard finite-difference scheme (44), $U_{i, j}^{n+1}$ and $V_{i, j}^{n+1}$ can be written as

$$
\left\{\begin{array}{l}
U_{i, j}^{n+1}=\frac{\phi(\Delta t)\left[\frac{U_{i+1, j}^{n}-2 U_{i, j}^{n}+U_{i-1, j}^{n}}{R \psi(\Delta x)^{2}}+\frac{U_{i, j+1}^{n}-2 U_{i, j}^{n}+U_{i, j-1}^{n}}{R \psi(\Delta y)^{2}}-V_{i, j}^{n} \frac{U_{i, j+1}^{n}-U_{i, j-1}^{n}}{2 \psi(\Delta y)}\right]+U_{i, j}^{n}}{\left(\phi(\Delta t) \frac{U_{i+1, j}^{n}-U_{i-1, j}^{n}}{2 \psi(\Delta x)}+1\right)}, \\
V_{i, j}^{n+1}=\frac{\varphi(\Delta t)\left[\frac{V_{i+1, j}^{n}-2 V_{i, j}^{n}+V_{i-1, j}^{n}}{R \psi(\Delta x)^{2}}+\frac{V_{i, j+1}^{n}-2 V_{i, j}^{n}+v_{i, j-1}^{n}}{R \psi(\Delta y)^{2}}-U_{i, j}^{n} \frac{V_{i+1, j}^{n}-V_{i-1, j}^{n}}{2 \psi(\Delta x)}\right]+V_{i, j}^{n}}{\left(\varphi(\Delta t) \frac{V_{i, j+1}^{n}-V_{i, j-1}^{n}}{2 \psi(\Delta y)}+1\right)} .
\end{array}\right.
$$

We have two different initial-boundary conditions in the following examples.

Example 1 The computational domain has been taken as $\Omega=\{(x, y): 0 \leq x \leq 1,0 \leq y \leq 1\}$ and the Burgers equations (45) are taken with the initial conditions

$$
\begin{cases}u(x, y, 0)=\frac{3}{4}-\frac{1}{4\left(1+e^{\frac{R}{32}(4 y-4 x)}\right)}, & 0 \leq x \leq 1,0 \leq y \leq 1 \\ v(x, y, 0)=\frac{3}{4}+\frac{1}{4\left(1+e^{\frac{R}{32}(4 y-4 x)}\right)}, & 0 \leq x \leq 1,0 \leq y \leq 1\end{cases}
$$

and the boundary conditions

$$
\begin{cases}u(0, y, t)=\frac{3}{4}-\frac{1}{4\left(1+e^{\frac{R}{32}(4 y-t)}\right)}, & 0 \leq y \leq 0.5, t>0, \\ u(1, y, t)=\frac{3}{4}-\frac{1}{4\left(1+e^{\frac{R}{32}(4 y-4-t)}\right)}, & 0 \leq y \leq 0.5, t>0, \\ v(0, y, t)=\frac{3}{4}+\frac{1}{4\left(1+e^{\frac{R}{32}(4 y-t)}\right)}, & 0 \leq y \leq 0.5, t>0, \\ v(1, y, t)=\frac{3}{4}+\frac{1}{4\left(1+e^{\frac{R}{32}(4 y-4-t)}\right)}, & 0 \leq y \leq 0.5, t>0, \\ \left.u(x, 0, t)=\frac{3}{4}-\frac{1}{4\left(1+e^{\frac{R}{32}}(-4 x-t)\right.}\right) & 0 \leq x \leq 0.5, t>0, \\ u(x, 1, t)=\frac{3}{4}-\frac{1}{4\left(1+e^{\frac{R}{32}}(4-4 x-t)\right.}, & 0 \leq x \leq 0.5, t>0, \\ v(x, 0, t)=\frac{3}{4}+\frac{1}{4\left(1+e^{\frac{R}{32}(-4 x-t)}\right)}, & 0 \leq x \leq 0.5, t>0, \\ v(x, 1, t)=\frac{3}{4}-\frac{1}{4\left(1+e^{\frac{R}{32}(4-4 x-t)}\right)}, & 0 \leq x \leq 0.5, t>0,\end{cases}
$$

where $u(x, y, t)$ and $v(x, y, t)$ are taken from the analytical solutions (2).

Base on the initial-boundary conditions, the numerical initial-boundary conditions can be written as

$$
\begin{cases}U_{i, j}^{0}=\frac{3}{4}-\frac{1}{4\left(1+e^{\frac{R}{32}\left(4 y_{j}-4 x_{i}\right)},\right.}, & i=0,1, \ldots, I, j=0,1, \ldots, J \\ V_{i, j}^{0}=\frac{3}{4}+\frac{1}{4\left(1+e^{\frac{R}{32}\left(4 y_{j}-4 x_{i}\right)}\right)}, & i=0,1, \ldots, I, j=0,1, \ldots, J \\ U_{0, j}^{n}=\frac{3}{4}-\frac{1}{4\left(1+e^{\frac{R}{32}\left(4 y_{j}-t_{n}\right)}\right)}, & j=0,1, \ldots, J, n=0,1, \ldots, \\ U_{I, j}^{n}=\frac{3}{4}-\frac{1}{4\left(1+e^{\frac{R}{32}\left(4 y_{j}-4-t_{n}\right)}\right)}, & j=0,1, \ldots, J, n=0,1, \ldots \\ V_{0, j}^{n}=\frac{3}{4}+\frac{1}{4\left(1+e^{\frac{R}{32}\left(4 y_{j}-t_{n}\right)}\right)}, & j=0,1, \ldots, J, n=0,1, \ldots \\ V_{I, j}^{n}=\frac{3}{4}+\frac{1}{4\left(1+e^{\frac{R}{32}\left(4 y_{j}-4-t_{n}\right)}\right)}, & j=0,1, \ldots, J, n=0,1, \ldots \\ U_{i, 0}^{n}=\frac{3}{4}-\frac{1}{4\left(1+e^{\frac{R}{32}\left(-4 x_{i}-t_{n}\right)}\right)}, & i=0,1, \ldots, I, n=0,1, \ldots \\ U_{i, J}^{n}=\frac{3}{4}-\frac{1}{4\left(1+e^{\frac{R}{32}\left(4-4 x_{i}-t_{n}\right)}\right)}, & i=0,1, \ldots, I, n=0,1, \ldots \\ V_{i, 0}^{n}=\frac{3}{4}+\frac{1}{4\left(1+e^{\frac{R}{32}\left(-4 x_{i}-t_{n}\right)}\right)}, & i=0,1, \ldots, I, n=0,1, \ldots \\ V_{i, J}^{n}=\frac{3}{4}-\frac{1}{4\left(1+e^{\frac{R}{32}\left(4-4 x_{i}-t_{n}\right)}\right)}, & i=0,1, \ldots, I, n=0,1, \ldots\end{cases}
$$


Table 1 The numerical results of first scheme (46) and second scheme (47) for $u$ with the exact solution at $t=0.01, t=0.5$ and $t=2$ with $t=0.0001$, and $R=10$

\begin{tabular}{|c|c|c|c|c|c|c|c|c|c|}
\hline \multirow[t]{2}{*}{$(x, y)$} & \multicolumn{3}{|l|}{$t=0.01$} & \multicolumn{3}{|l|}{$t=0.5$} & \multicolumn{3}{|l|}{$t=2$} \\
\hline & First & Second & Exact & First & Second & Exact & First & Second & Exact \\
\hline$(0.1,0.1)$ & 0.62480 & 0.62481 & 0.62480 & 0.61522 & 0.61528 & 0.61525 & 0.58713 & 0.58718 & 0.58716 \\
\hline$(0.5,0.1)$ & 0.59419 & 0.59421 & 0.59420 & 0.58533 & 0.58544 & 0.58540 & 0.56120 & 0.56130 & 0.56127 \\
\hline$(0.9,0.1)$ & 0.56708 & 0.56708 & 0.56708 & 0.55979 & 0.55986 & 0.55983 & 0.54108 & 0.54114 & 0.54113 \\
\hline$(0.3,0.3)$ & 0.62480 & 0.62481 & 0.62480 & 0.61512 & 0.61538 & 0.61525 & 0.58701 & 0.58725 & 0.58716 \\
\hline$(0.7,0.3)$ & 0.59419 & 0.59421 & 0.59420 & 0.58522 & 0.58551 & 0.58540 & 0.56108 & 0.56136 & 0.56127 \\
\hline$(0.1,0.5)$ & 0.65543 & 0.65544 & 0.65543 & 0.64622 & 0.64635 & 0.64628 & 0.61712 & 0.61726 & 0.61720 \\
\hline$(0.5,0.5)$ & 0.62480 & 0.62481 & 0.62480 & 0.61503 & 0.61546 & 0.61525 & 0.58688 & 0.58734 & 0.58716 \\
\hline$(0.9,0.5)$ & 0.59419 & 0.59421 & 0.59420 & 0.58523 & 0.58551 & 0.58540 & 0.56108 & 0.56136 & 0.56127 \\
\hline$(0.3,0.7)$ & 0.65543 & 0.65544 & 0.65543 & 0.64612 & 0.64648 & 0.64628 & 0.61698 & 0.61739 & 0.61729 \\
\hline$(0.7,0.7)$ & 0.62480 & 0.62481 & 0.62480 & 0.61500 & 0.61550 & 0.61525 & 0.58680 & 0.58740 & 0.58716 \\
\hline$(0.1,0.9)$ & 0.68261 & 0.68262 & 0.68261 & 0.67478 & 0.67488 & 0.67481 & 0.64811 & 0.64823 & 0.64817 \\
\hline$(0.5,0.9)$ & 0.65543 & 0.65544 & 0.65543 & 0.64613 & 0.64647 & 0.64628 & 0.61696 & 0.61740 & 0.61720 \\
\hline$(0.9,0.9)$ & 0.62480 & 0.62481 & 0.62480 & 0.61512 & 0.61537 & 0.61525 & 0.58697 & 0.58728 & 0.58716 \\
\hline
\end{tabular}

Table 2 The numerical results of first scheme (46) and second scheme (47) for $v$ with the exact solution at $t=0.01, t=0.5$ and $t=2$ with $t=0.0001$, and $R=10$

\begin{tabular}{|c|c|c|c|c|c|c|c|c|c|}
\hline \multirow[t]{2}{*}{$(x, y)$} & \multicolumn{3}{|l|}{$t=0.01$} & \multicolumn{3}{|l|}{$t=0.5$} & \multicolumn{3}{|l|}{$t=2$} \\
\hline & First & Second & Exact & First & Second & Exact & First & Second & Exact \\
\hline$(0.1,0.1)$ & 0.87519 & 0.87519 & 0.87520 & 0.88471 & 0.88472 & 0.88475 & 0.91280 & 0.91282 & 0.91284 \\
\hline$(0.5,0.1)$ & 0.90579 & 0.90579 & 0.90580 & 0.91454 & 0.91456 & 0.91460 & 0.93866 & 0.93870 & 0.93873 \\
\hline$(0.9,0.1)$ & 0.93291 & 0.93292 & 0.93292 & 0.94011 & 0.94014 & 0.94016 & 0.95883 & 0.95886 & 0.95887 \\
\hline$(0.3,0.3)$ & 0.87519 & 0.87519 & 0.87520 & 0.88461 & 0.88462 & 0.88475 & 0.91268 & 0.91275 & 0.91284 \\
\hline$(0.7,0.3)$ & 0.90579 & 0.90579 & 0.90580 & 0.91442 & 0.91449 & 0.91460 & 0.93853 & 0.93864 & 0.93873 \\
\hline$(0.1,0.5)$ & 0.84456 & 0.84456 & 0.84457 & 0.85366 & 0.85365 & 0.85372 & 0.88272 & 0.88274 & 0.88280 \\
\hline$(0.5,0.5)$ & 0.87519 & 0.87519 & 0.87520 & 0.88452 & 0.88454 & 0.88475 & 0.91255 & 0.91266 & 0.91284 \\
\hline$(0.9,0.5)$ & 0.90579 & 0.90579 & 0.90580 & 0.91444 & 0.91449 & 0.91460 & 0.93854 & 0.93864 & 0.93873 \\
\hline$(0.3,0.7)$ & 0.84456 & 0.84456 & 0.84457 & 0.85357 & 0.85352 & 0.85372 & 0.88257 & 0.88261 & 0.88280 \\
\hline$(0.7,0.7)$ & 0.87519 & 0.87519 & 0.87520 & 0.88449 & 0.88450 & 0.88475 & 0.91247 & 0.91260 & 0.91284 \\
\hline$(0.1,0.9)$ & 0.81739 & 0.81738 & 0.81739 & 0.82515 & 0.82512 & 0.82519 & 0.85178 & 0.85177 & 0.85183 \\
\hline$(0.5,0.9)$ & 0.84456 & 0.84456 & 0.84457 & 0.85358 & 0.85353 & 0.85372 & 0.88256 & 0.88260 & 0.88280 \\
\hline$(0.9,0.9)$ & 0.87519 & 0.87519 & 0.87520 & 0.88462 & 0.88463 & 0.88475 & 0.91266 & 0.91272 & 0.91284 \\
\hline
\end{tabular}

The numerical simulation is performed using a uniform grid $20 \times 20$, with a mesh width $\Delta x=\Delta y=0.05$. In Tables 1-2 are the numerical results for $u$ and $v$ compared with the exact solution at $t=0.01, t=0.5$ and $t=2$ with $\Delta t=0.0001$, and $R=10$. And Figures 1-12 show the numerical errors for $u$ and $v$ at $t=0.01, t=0.5$ and $t=2$ with $\Delta t=0.0001$, and $R=10$. From Tables 1-2 and Figures 1-6, we can see that our explicit nonstandard finite-difference schemes behave in a good manner.

Example 2 The computational domain has been taken as $\Omega=\{(x, y): 0 \leq x \leq 0.5,0 \leq y \leq$ $0.5\}$ and the Burgers equations (45) are taken with the initial conditions

$$
\begin{cases}u(x, y, 0)=\sin (\pi x)+\cos (\pi y), & 0 \leq x \leq 0.5,0 \leq y \leq 0.5 \\ v(x, y, 0)=x+y, & 0 \leq x \leq 0.5,0 \leq y \leq 0.5\end{cases}
$$




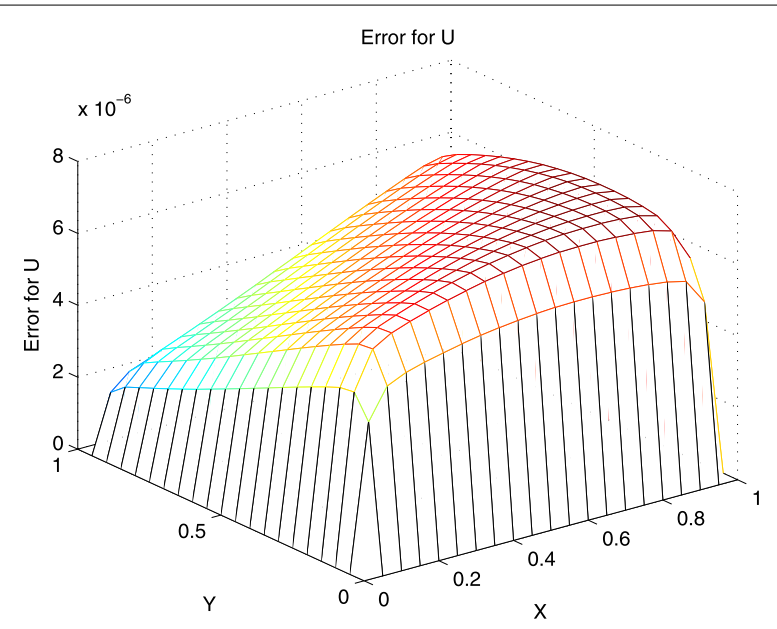

Figure 1 Error of $U_{i j}^{n}$ for scheme (46) of $u$ with $R=10$ at $t=0.01$.

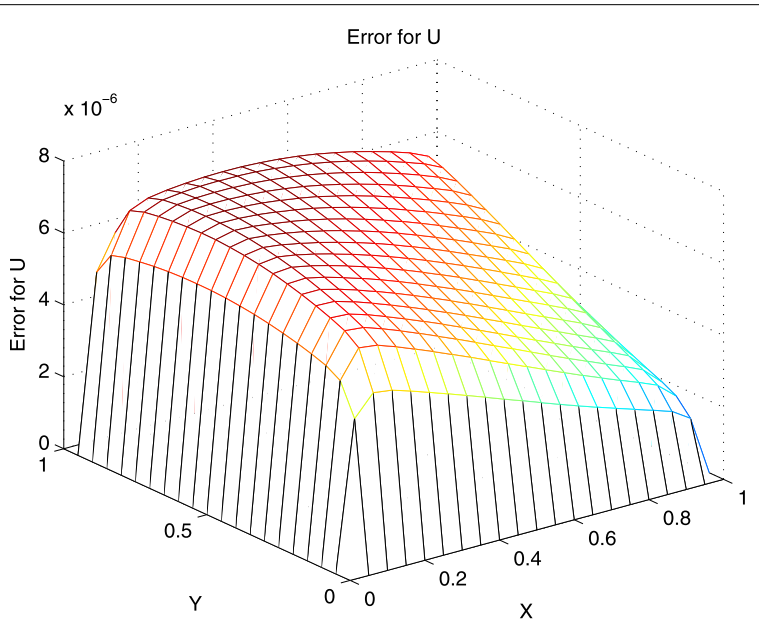

Figure 2 Error of $U_{i j}^{n}$ for scheme (47) of $u$ with $R=10$ at $t=0.01$.

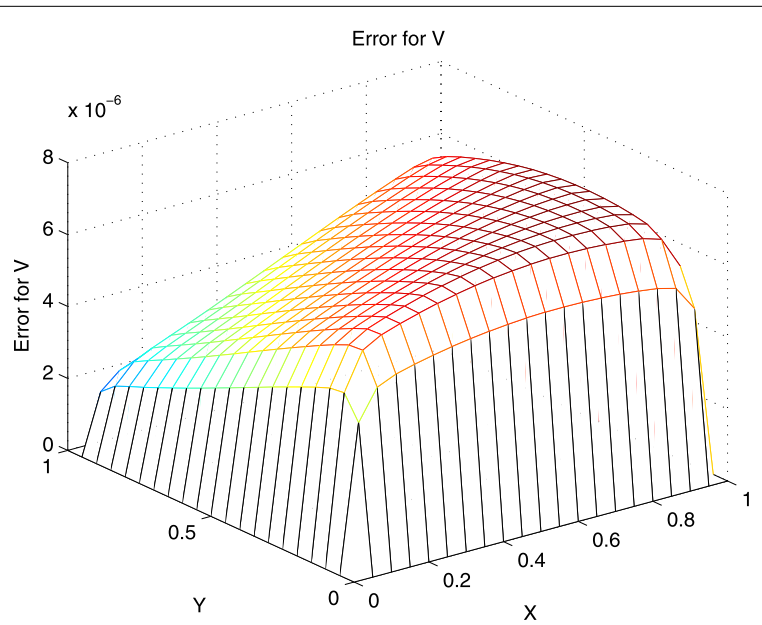

Figure 3 Error of $V_{i j}^{n}$ for scheme (46) of $v$ with $R=10$ at $t=0.01$. 
Chang et al. Advances in Difference Equations 2014, 2014:122

Page 18 of 24

http://www.advancesindifferenceequations.com/content/2014/1/122

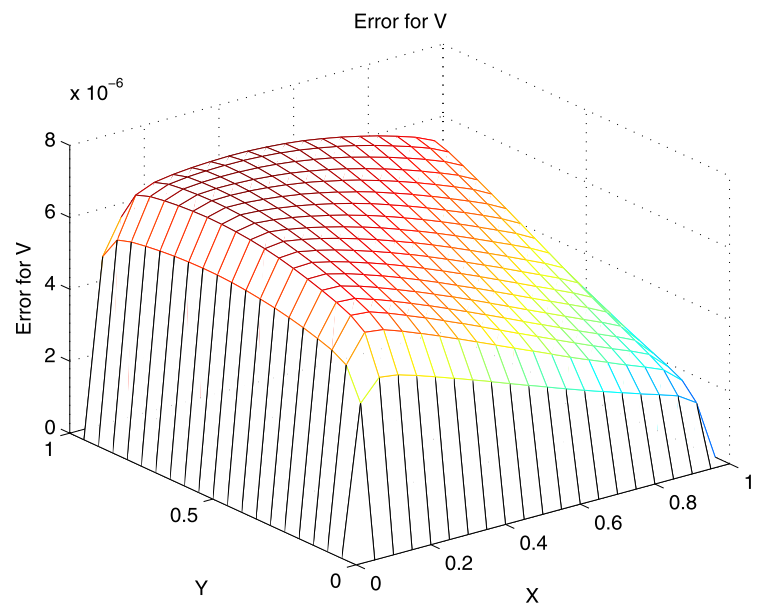

Figure 4 Error of $V_{i j}^{n}$ for scheme (47) of $v$ with $R=10$ at $t=0.01$.

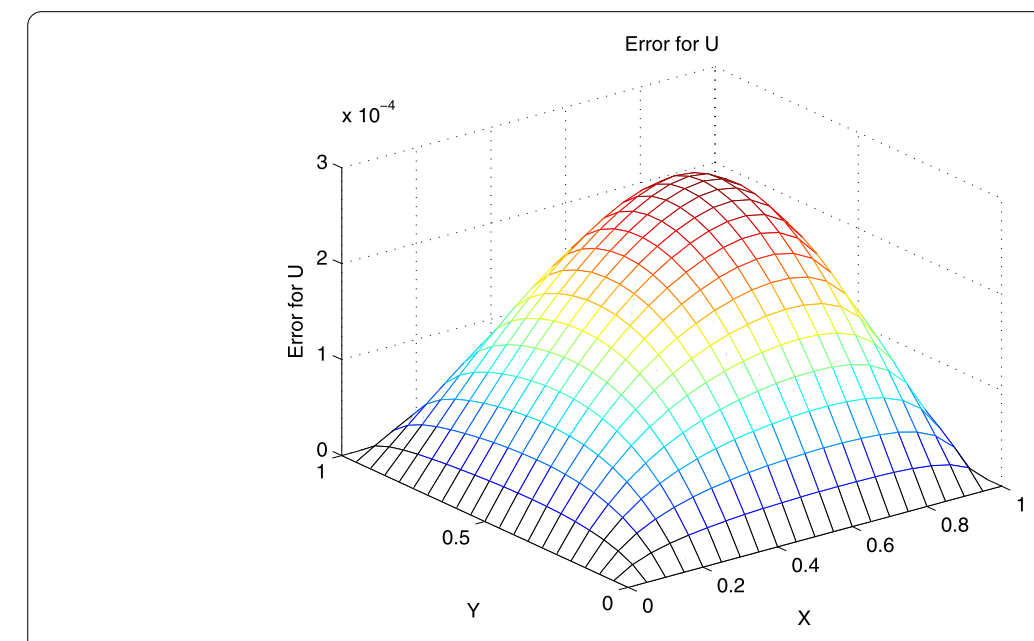

Figure 5 Error of $U_{i j}^{n}$ for scheme (46) of $u$ with $R=10$ at $t=0.5$.

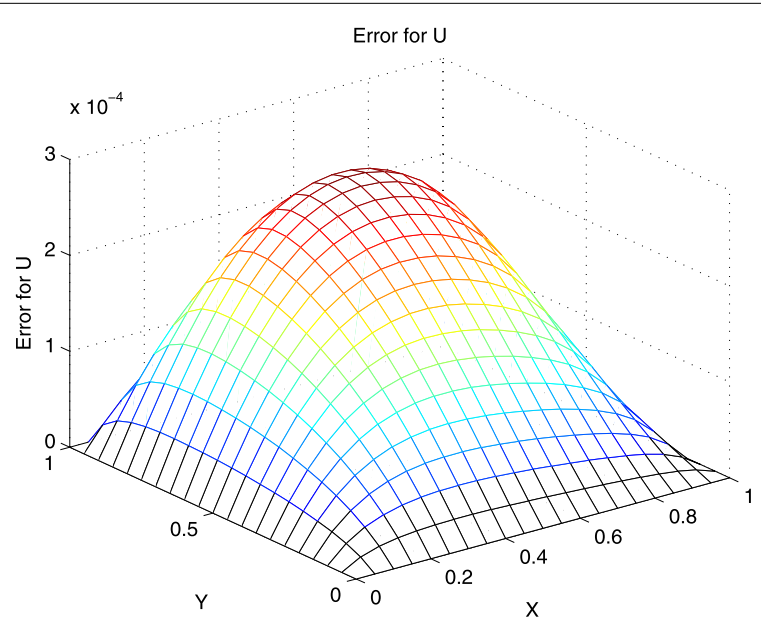

Figure 6 Error of $U_{i j}^{n}$ for scheme (47) of $u$ with $R=10$ at $t=0.5$. 
Chang et al. Advances in Difference Equations 2014, 2014:122

Page 19 of 24

http://www.advancesindifferenceequations.com/content/2014/1/122

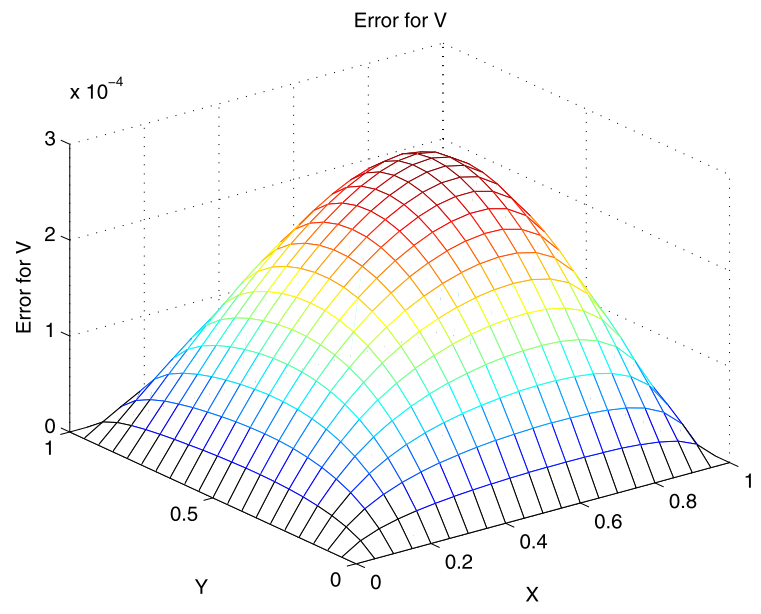

Figure 7 Error of $V_{i j}^{n}$ for scheme (46) of $v$ with $R=10$ at $t=0.5$.

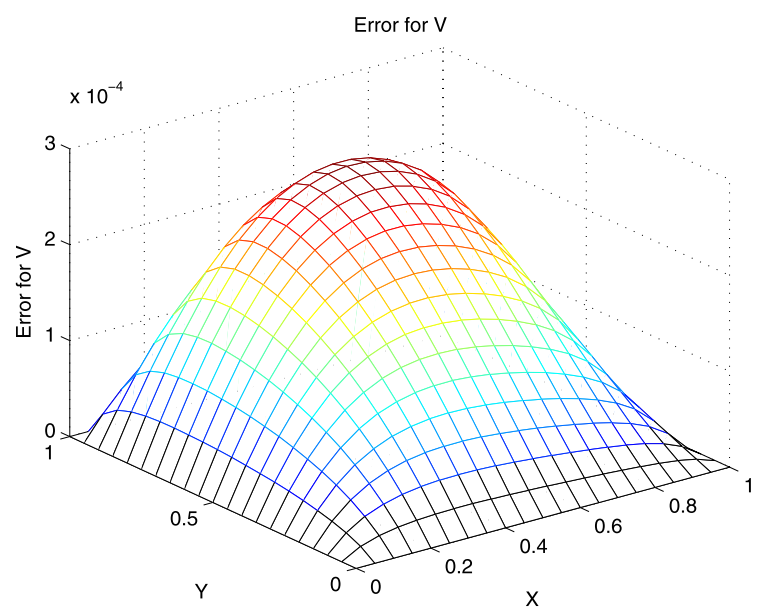

Figure 8 Error of $V_{i j}^{n}$ for scheme (47) of $v$ with $R=10$ at $t=0.5$.

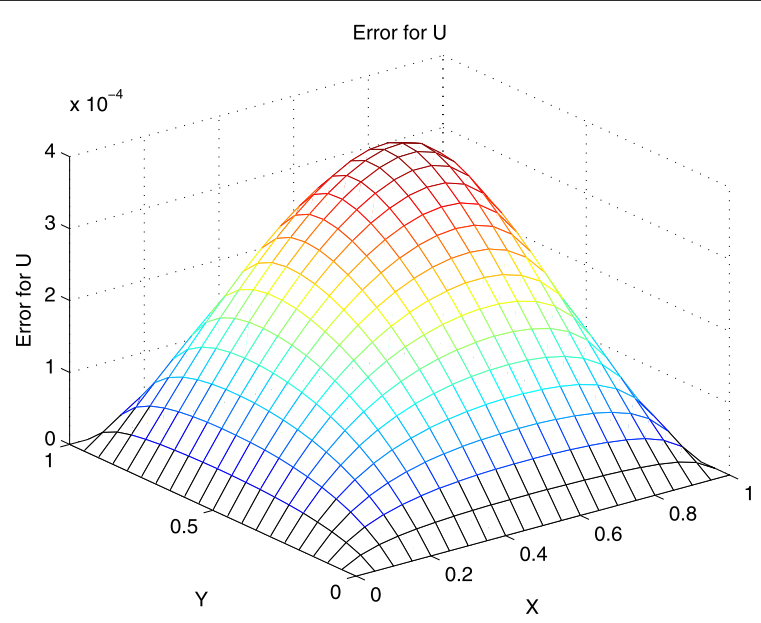

Figure 9 Error of $U_{i j}^{n}$ for scheme (46) of $u$ with $R=10$ at $t=2$. 
Chang et al. Advances in Difference Equations 2014, 2014:122

Page 20 of 24

http://www.advancesindifferenceequations.com/content/2014/1/122

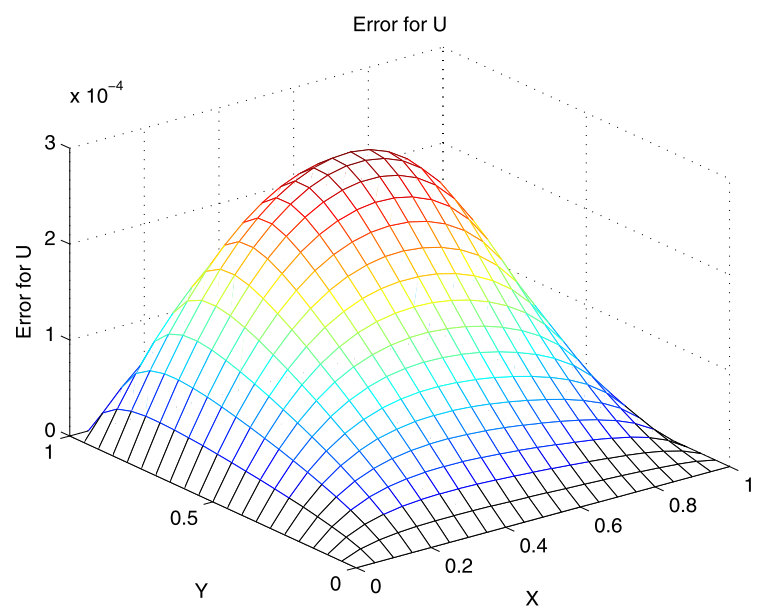

Figure 10 Error of $U_{i j}^{n}$ for scheme (47) of $u$ with $R=10$ at $t=2$.

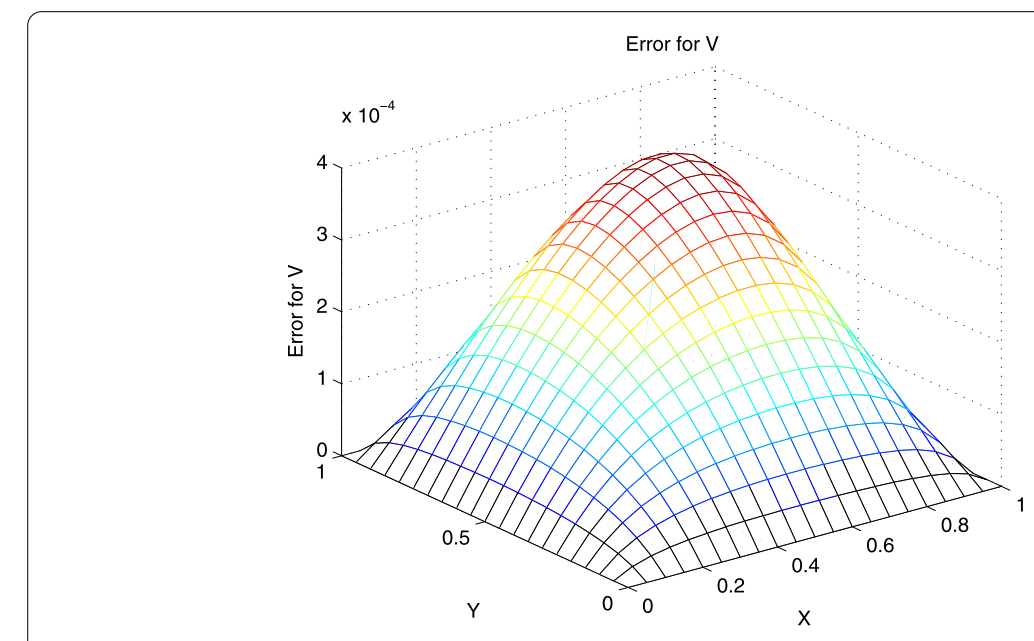

Figure 11 Error of $V_{i j}^{n}$ for scheme (46) of $v$ with $R=10$ at $t=2$.

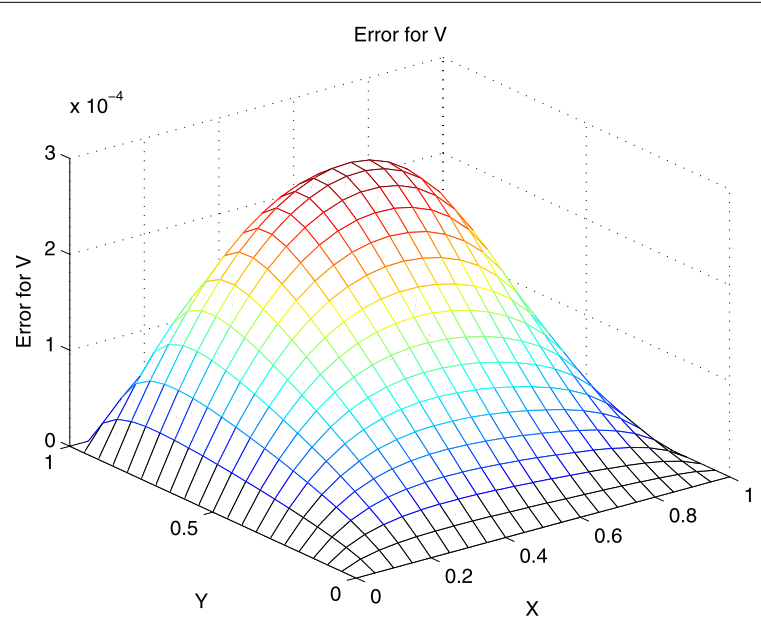

Figure 12 Error of $V_{i j}^{n}$ for scheme (47) of $v$ with $R=10$ at $t=2$. 
Table 3 The numerical results of first scheme (46) and second scheme (47) for $u$ and $v$ with Vineet, Bahadir and Jain's solutions at $t=0.625$ with $t=0.0001$, and $R=50,20 \times 20$ grid

\begin{tabular}{|c|c|c|c|c|c|c|c|c|c|c|}
\hline \multirow[t]{2}{*}{$(x, y)$} & \multicolumn{5}{|c|}{ Computed values of $U$} & \multicolumn{5}{|c|}{ Computed values of $\boldsymbol{V}$} \\
\hline & First & econd & Srivastava & Bahadir & Jain & First & Second & Srivastava & Bahadir & Jain \\
\hline & 0.95754 & 0.96925 & 0.97146 & 0.96688 & 0.97258 & 0.09364 & 0.09774 & 0.098688 & 0.09824 & 0.09773 \\
\hline & & & & & & & & & & \\
\hline & & & & & & & & & & 0.16660 \\
\hline & & & & & & & & & & 0.17397 \\
\hline & 0.67190 & 0.66426 & 0.66 & 0.66019 & 0.66318 & 0.26263 & 0.26355 & 0.26 & 0.26261 & 0.26294 \\
\hline 0.3$)$ & 0.76523 & 0.77524 & 0.77233 & 0.76932 & 0.77030 & 0.22205 & 0.22650 & 0.22655 & 0.22576 & 0.22463 \\
\hline$(0.2,0.4)$ & 0.58001 & 0.58333 & 0.58181 & 0.57966 & 0.58070 & 0.30996 & 0.32554 & 0.32851 & 0.32745 & 0.32402 \\
\hline$(0.4,0.4)$ & 0.70944 & 0.76524 & 0.75862 & 0.75678 & 0.74435 & 0.30151 & 0.32120 & 0.32502 & 0.32441 & 0.31822 \\
\hline
\end{tabular}

and the boundary conditions

$$
\begin{cases}u(0, y, t)=\cos (\pi y), & 0 \leq y \leq 0.5, t>0 \\ u(0.5, y, t)=1+\cos (\pi y), & 0 \leq y \leq 0.5, t>0 \\ v(0, y, t)=y, & 0 \leq y \leq 0.5, t>0 \\ v(0.5, y, t)=0.5+y, & 0 \leq y \leq 0.5, t>0 \\ u(x, 0, t)=1+\sin (\pi x), & 0 \leq x \leq 0.5, t>0 \\ u(x, 0.5, t)=\sin (\pi x), & 0 \leq x \leq 0.5, t>0 \\ v(x, 0, t)=x, & 0 \leq x \leq 0.5, t>0 \\ v(x, 0.5, t)=x+0.5, & 0 \leq x \leq 0.5, t>0\end{cases}
$$

The numerical initial-boundary conditions are described as follows:

$$
\begin{cases}U_{i, j}^{0}=\sin \left(\pi x_{i}\right)+\cos \left(\pi y_{j}\right), & i=0,1, \ldots, I, j=0,1, \ldots, J, \\ V_{i, j}^{0}=x_{i}+y_{j}, & i=0,1, \ldots, I, j=0,1, \ldots, J, \\ U_{0, j}^{n}=\cos \left(\pi y_{j}\right), & j=0,1, \ldots, J, n=0,1, \ldots, \\ U_{I, j}^{n}=1+\cos \left(\pi y_{j}\right), & j=0,1, \ldots, J, n=0,1, \ldots, \\ V_{0, j}^{n}=y_{j}, & j=0,1, \ldots, J, n=0,1, \ldots, \\ V_{I, j}^{n}=0.5+y_{j}, & j=0,1, \ldots, J, n=0,1, \ldots, \\ U_{i, 0}^{n}=1+\sin \left(\pi x_{i}\right), & i=0,1, \ldots, I, n=0,1, \ldots, \\ U_{i, J}^{n}=\sin \left(\pi x_{i}\right), & i=0,1, \ldots, I, n=0,1, \ldots, \\ V_{i, 0}^{n}=x_{i}, & i=0,1, \ldots, I, n=0,1, \ldots, \\ V_{i, J}^{n}=x_{i}+0.5, & i=0,1, \ldots, I, n=0,1, \ldots\end{cases}
$$

The numerical computations for the two schemes (47) and (48) are performed using $20 \times 20$ grids and $t=0.0001$. The steady state solutions for $R=50$ are obtained at $t=$ 0.625. Table 3 is the numerical steady state solutions for our methods compared with the methods of Srivastava et al. [1], Bahadir [3], and Jain and Holla [4]. From Table 3, it can be seen that numerical results obtained by our methods are in good agreement with Srivastava, Bahadir and Jain's methods. Perspective views of $u$ and $v$ for $R=50$ at $t=0.0001$ are given in Figures 13-16.

\section{Conclusions}

This paper presents exact finite-difference schemes and nonstandard finite-difference schemes for the Burgers equations with the form (1) based upon the analytic solution. 


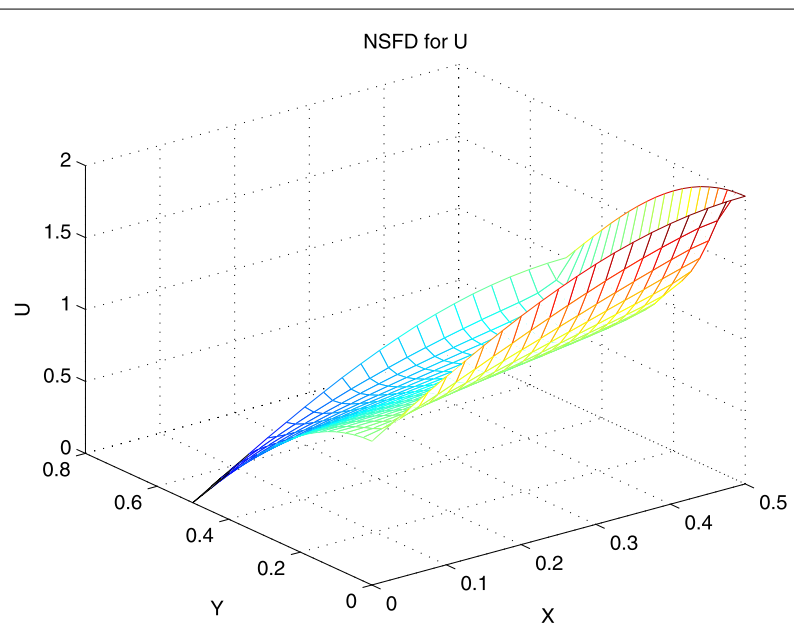

Figure 13 The computed value for scheme (46) of $u$ for $R=50$ at $t=0.625$.

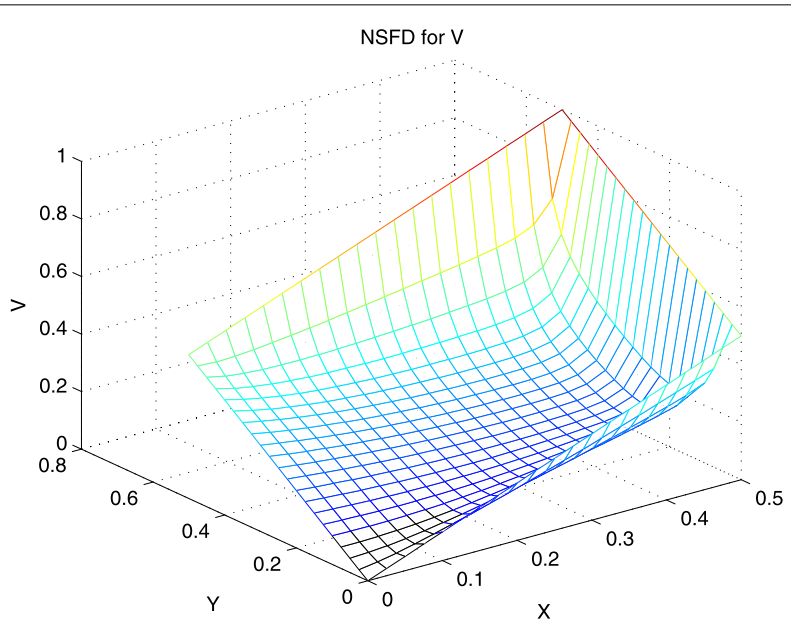

Figure 14 The computed value for scheme (46) of $v$ for $R=50$ at $t=0.625$.

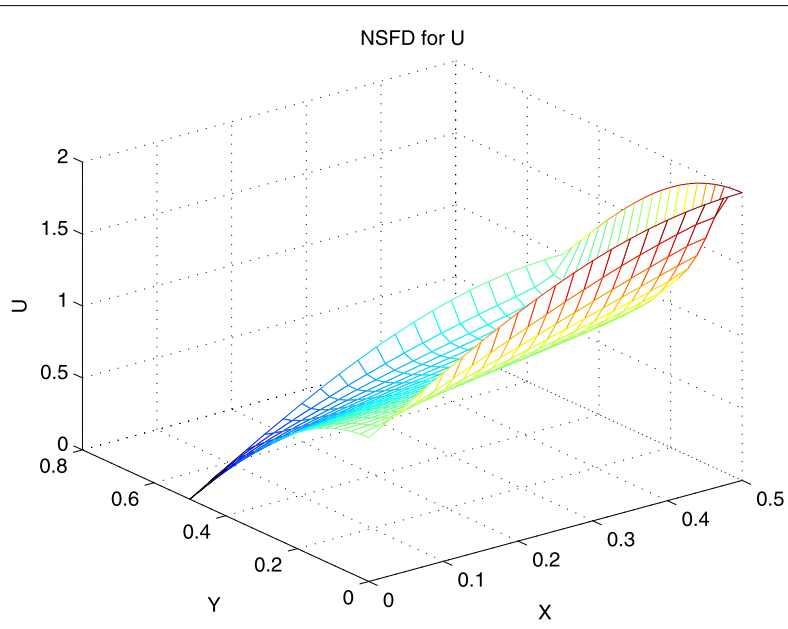

Figure 15 The computed value for scheme (47) of $u$ for $R=50$ at $t=0.625$. 


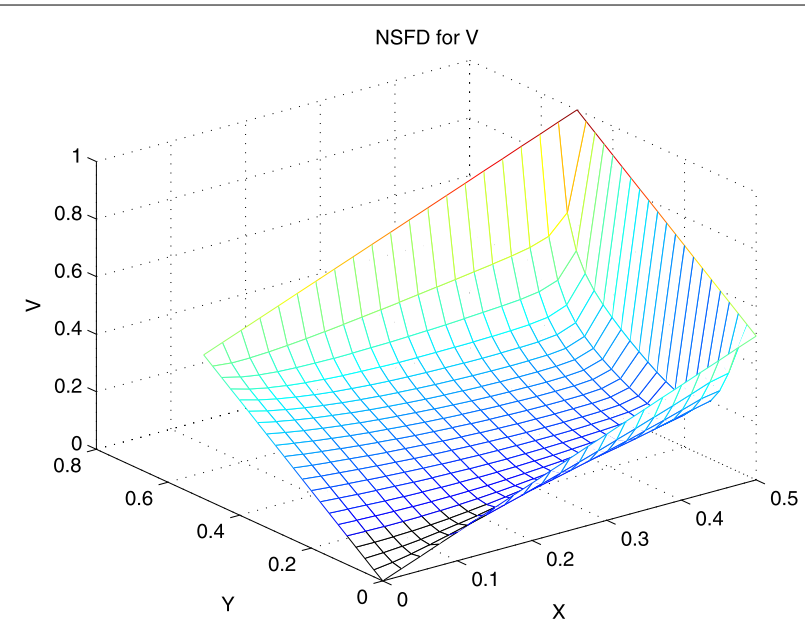

Figure 16 The computed value for scheme (47) of $v$ for $R=50$ at $t=0.625$.

Our nonstandard finite-difference schemes are explicit. The numerical simulations verify that the nonstandard finite-difference schemes are efficient and accurate.

\section{Competing interests}

The authors declare that there is no conflict of interests.

\section{Authors' contributions}

The authors declare that the study was realized in collaboration with the same responsibility. All authors read and approved the final manuscript.

\section{Acknowledgements}

This work is supported by the NNSF of China (No. 11271101) and the NNSF of Shandong Province (No. ZR2012AQ027).

Received: 3 February 2014 Accepted: 12 April 2014 Published: 06 May 2014

\section{References}

1. Srivastava, VK, Singh, S, Awasthi, MK: Numerical solutions of coupled Burgers' equations by an implicit finite difference scheme. AIP Adv. 3(8), 082131 (2013)

2. Fletcher, CAJ: Generating exact solutions of the two-dimensional Burgers' equation. Int. J. Numer. Methods Fluids 3(3), 213-216 (1983)

3. Bahadir, AR: A fully implicit finite-difference scheme for two-dimensional Burgers' equation. Appl. Math. Comput. 137(1), 131-137 (2003)

4. Jain, PC, Holla, DN: Numerical solution of coupled Burgers' equations. Int. J. Non-Linear Mech. 13(4), 213-222 (2003)

5. Srivastava, VK, Tamsir, M, Bhardwaj, U, Sanyasiraju, YVSS: Crank-Nicolson scheme for numerical solutions of two dimensional coupled Burgers' equations. Int. J. Sci. Eng. Res. 2(5), 1-6 (2011)

6. Tamsir, M, Srivastava, VK: A semi-implicit finite-difference approach for two-dimensional coupled Burgers' equations. Int. J. Sci. Eng. Res. 2, 1-6 (2011)

7. Srivastava, VK, Tamsir, M: Crank-Nicolson semi-implicit approach for numerical solutions of two-dimensional coupled nonlinear Burgers' equations. Int. J. Appl. Mech. Eng. 17(2), 571-581 (2012)

8. Kweyu, MC, Manyonge, WA, Koross, A, Ssemaganda, V: Numerical solutions of the Burgers' system in two dimensions under varied initial and boundary conditions. Appl. Math. Sci. 6(113), 5603-5615 (2012)

9. Mickens, RE, Oyedeji, K, Rucker, S: Exact finite difference scheme for second-order linear odes having constant coefficients. J. Sound Vib. 287(4), 1052-1056 (2005)

10. Rucker, S: Exact finite difference scheme for an advection-reaction equation. J. Differ. Equ. Appl. 9(11), 1007-1013 (2003)

11. Roeger, LW, Mickens, RE: Exact finite-difference schemes for first order differential equations having three distinct fixed-points. J. Differ. Equ. Appl. 13(12), 1179-1185 (2007)

12. Roeger, LW: Exact finite-difference schemes for two-dimensional linear systems with constant coefficients. J. Comput. Appl. Math. 219(1), 102-109 (2008)

13. Roeger, LW: Exact nonstandard finite-difference methods for a linear system - the case of centers. J. Differ. Equ. Appl. 14(4), 381-389 (2008)

14. Zhang, L, Wang, L, Ding, X: Exact finite difference scheme and nonstandard finite difference scheme for Burgers and Burgers-Fisher equations. J. Appl. Math. 2014, Article ID 597926 (2014) 
10.1186/1687-1847-2014-122

Cite this article as: Zhang et al.: Exact finite-difference scheme and nonstandard finite-difference scheme for coupled Burgers equation. Advances in Difference Equations 2014, 2014:122

Submit your manuscript to a SpringerOpen ${ }^{\circ}$ journal and benefit from:

- Convenient online submission

Rigorous peer review

- Immediate publication on acceptance

Open access: articles freely available online

- High visibility within the field

- Retaining the copyright to your article

Submit your next manuscript at $\boldsymbol{s p r i n g e r o p e n . c o m ~}$ 